\title{
Axion decay constants at special points in type II string theory
}

\author{
Masaki Honda, Akane Oikawa and Hajime Otsuka \\ Department of Physics, Waseda University, \\ Tokyo 169-8555, Japan \\ E-mail: yakkuru_111@ruri.waseda.jp, a.oikawa@aoni.waseda.jp, \\ h.otsuka@aoni.waseda.jp
}

ABSTRACT: We propose the mechanism to disentangle the decay constant of closed string axion from the string scale in the framework of type II string theory on Calabi-Yau manifold. We find that the quantum and geometrical corrections in the prepotential that arise at some special points in the moduli space widen the window of axion decay constant. In particular, around the small complex structure points, the axion decay constant becomes significantly lower than the string scale. We also discuss the moduli stabilization leading to the phenomenologically attractive low-scale axion decay constant.

KEYWORDs: Strings and branes phenomenology

ARXIV EPRINT: 1608.08372 


\section{Contents}

1 Introduction 1

2 Decay constant of closed string axion 3

3 Axion decay constant around the special point $\quad 6$

3.1 SCS point 6

3.1.1 $\alpha_{1} \neq \alpha_{2} \neq \alpha_{3} \neq \alpha_{4}$

$\begin{array}{ll}3.1 .2 & \alpha_{1} \neq \alpha_{2} \neq \alpha_{3}=\alpha_{4}\end{array}$

3.1.3 $\alpha_{1}=\alpha_{2} \neq \alpha_{3}=\alpha_{4} \quad 13$

3.1.4 $\alpha_{1}=\alpha_{2}=\alpha_{3}=\alpha_{4} \quad 14$

$\begin{array}{lll}3.2 & \text { Conifold point } & 15\end{array}$

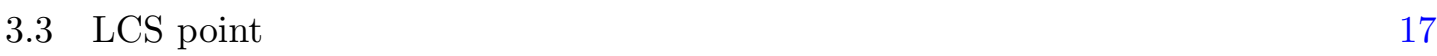

$\begin{array}{lll}3.3 .1 & \text { Single axion } & 19\end{array}$

$\begin{array}{lll}3.3 .2 & \text { Multiple axions } & 20\end{array}$

4 Massless axion and moduli stabilization $\quad 22$

5 Conclusion $\quad 25$

\section{Introduction}

An axion, or axion-like particle, is phenomenologically and cosmologically attractive particle to explain the origin of tiny strong CP phase in the standard model [1], the current dark matter abundance [2-4] and the origin of cosmological microwave background through the inflation mechanism. The consistent theory of quantum gravity such as the string theory also predicts the existence of axion particles through the dimensional reduction of the higher-dimensional vector and tensor fields associated with the internal cycles of extradimensional space.

In particular, the QCD axion decay constant should be within the range, $10^{9-12} \mathrm{GeV}$ by the observation of supernova (SN) 1987A $[5,6]$ and dark matter abundance observed by Planck [7] with $\mathcal{O}(1)$ initial misalignment angle.(For a review, see, e.g., ref. [8].) From the inflationary point of view, the decay constant of axion inflaton is severely constrained by the Planck data [7], e.g., $10^{18-19} \mathrm{GeV}$ for the natural inflation [9]. It is difficult to extract the above constrained axion decay constant from the string theory. The authors of refs. [10-12] showed that decay constant of closed string axion in the string theory is typically around $10^{16-17} \mathrm{GeV}$, since both the decay constant and the gauge coupling of visible sector are closely related through the volume of extra-dimensional space. When the visible sector lives on the localized cycle of extra-dimensional space, the decay constant of axion associated 
with the large volume cycle can be taken much smaller than Planck scale [13], which is achieved in so-called LARGE Volume Scenario (LVS) in type IIB string theory [14]. ${ }^{1}$ On the other hand, the larger axion decay constant is obtained by the inclusion of the quantum corrections to the volume of extra-dimensional space [18], one-loop corrections for the gauge couplings [19, 20], and the alignment mechanism in terms of multiple axions [21].

In this paper, we focus on Kähler (complex structure) moduli fields in type IIA (IIB) string theory on Calabi-Yau (CY) manifold. These moduli potentials receive the quantum (geometrical) corrections which are exactly calculated in the topological string theory. Recently, the authors of ref. [18] have showed that the axion decay constant including the instanton corrections has the maximum value in type IIA string on CY manifold with a few moduli fields around the large volume limit, which corresponds to the large complex structure (LCS) limit in type IIB string on mirror CY manifold. In this case, even if the volume of internal cycle is of $\mathcal{O}(1)$ in string units, such instanton corrections give the sizable effect. In this way, it motivates us to proceed to study the detail of the quantum and geometrical corrections for a decay constant of closed string axion around the several points of moduli spaces. In type IIB string theory on CY manifold, these geometrical and non-perturbative corrections are exactly obtained by solving the corresponding PicardFuchs differential equation for the period vector of CY manifold, which corresponds to the instanton corrections in type IIA side.

We in particular focus on the regular singular points involved in the Picard-Fuchs equation which are called as the special points of moduli space, such as the LCS point, conifold point, and small complex structure (SCS) point involving the Gepner point [22, 23]. These closed string axions then naturally appear around the special points in the low-energy effective theory, since the monodromy symmetries around special points allow the existence of axions in the moduli Kähler potential. Around these special points, we proceed to study the detail of the quantum and geometrical corrections for a decay constant of closed string axion. It is remarkable that in type IIB string theory, the decay constants of axions associated with the complex structure moduli are irrelevant to the volume of mirror CY manifold, i.e., the string scale, in comparison with those of Kähler moduli. As pointed out in refs. [24-26], it is interesting to discuss the phenomenology and cosmology of complex structure moduli.

The remaining of this paper is organized as follows. After briefly reviewing the Kähler potential on the basis of $\mathcal{N}=2$ special geometry in section 2, we first show how to define the axion particles around the special points of complex structure moduli space and corresponding decay constant. The axionic shift symmetries are then captured by the invariance of Kähler potential under the monodromy transformation at the special points. In section 3.1, we formulate the geometrical corrections for the period vector of CY manifold with an emphasis on the SCS point. It is then found that, in contrast to the previous studies, the decay constant of closed string axion associated with the complex structure modulus is taken much smaller than the string scale, and such a property is a common phenomena in typical one-parameter CY manifolds. Next, we proceed to estimate the

\footnotetext{
${ }^{1}$ The warped string compactification is also discussed in ref. [15]. See for other axions irrelevant with the internal cycle of extra-dimensional space in heterotic string [16] and type IIA string [17].
} 
axion decay constant around the conifold point in section 3.2 and LCS point in section 3.3. Finally, we comment on the moduli stabilization to generate the low-scale axion decay constants in section 4 . Section 5 is devoted to the conclusion.

\section{Decay constant of closed string axion}

Throughout this paper, we consider the low-energy effective theory of type II string theory on $\mathrm{CY}$ orientifold in which the closed string moduli are categorized into the axion-dilaton $\tau$, Kähler moduli $T$, and complex structure moduli $z$. (See for the construction of fourdimensional $\mathcal{N}=1$ effective theory on CY orientifold, e.g., ref. [27].)

First of all, we show the decay constant of axion associated with Kähler moduli in type IIB string theory on CY orientifold. ${ }^{2}$ In the framework of four-dimensional $\mathcal{N}=1$ supergravity action, the relevant Lagrangian density of closed string axion associated with the Kalb-Ramond field or Ramond-Ramond field is captured by

$$
\mathcal{L}=-K_{\rho \bar{\rho}} \partial_{\mu} \theta \partial^{\mu} \theta-\frac{1}{4 g^{2}} F^{\mu \nu} F_{\mu \nu}-\frac{\theta}{4} F^{\mu \nu} \tilde{F}_{\mu \nu},
$$

where $\theta(x)$ is the axion accompanied by the certain modulus field $\rho, K_{\rho \bar{\rho}}=\partial^{2} K / \partial \rho \partial \bar{\rho}$ is the second derivative of the Kähler potential $K$ with respect to $\rho$ and $\bar{\rho}, \mu, \nu$ denote the indices of Minkowski spacetime, and $\tilde{F}_{\mu \nu}$ is the dual gauge field strength of $F_{\mu \nu}$ in the visible or hidden sector with the gauge coupling $g$. Since the axion potential is non-perturbatively generated by breaking the higher-dimensional gauge symmetries to discrete one, the axion decay constant $f$ is extracted by canonically normalizing the axion as $\tilde{\theta}=\sqrt{2 K_{\rho \bar{\rho}}} \theta$,

$$
\mathcal{L}=-\frac{1}{2} \partial_{\mu} \tilde{\theta} \partial^{\mu} \tilde{\theta}-\frac{\tilde{\theta}}{32 \pi^{2} f} F^{\mu \nu} \tilde{F}_{\mu \nu}
$$

with

$$
f=\frac{\sqrt{2 K_{\rho \bar{\rho}}}}{8 \pi^{2}} .
$$

When $\rho$ corresponds the Kähler modulus $T$ of CY manifold $\mathcal{M}$ in the type IIB string theory, the classical modulus Kähler potential is characterized by the volume of CY manifold $\mathcal{V}$ in string units, i.e., $K=-2 M_{\mathrm{Pl}}^{2} \ln \mathcal{V}$ with $M_{\mathrm{Pl}}$ being the reduced Planck mass. ${ }^{3}$ This Kähler potential is valid in the large volume limit of CY manifold $\mathcal{V}=(T+\bar{T})^{3 / 2}>1$, and then the decay constant of axion $\operatorname{Im} T$ reads as

$$
f \simeq \frac{\sqrt{6}}{\mathcal{V}^{2 / 3}} \frac{M_{\mathrm{Pl}}}{8 \pi^{2}}
$$

For the closed string axion in the string theory, the (internal) volume of CY manifold is related to the value of gauge coupling. Thus, the observed value of gauge coupling in the

\footnotetext{
${ }^{2}$ It is straightforward to extend the following discussion to type IIA string theory or heterotic string with standard embedding.

${ }^{3}$ We use the reduced Planck unit $M_{\mathrm{Pl}}=2.4 \times 10^{18} \mathrm{GeV}=1$ unless otherwise specified.
} 
visible sector leads to the constrained axion decay constant around $10^{16-17} \mathrm{GeV}$ [12], which is the same order of the string scale

$$
M_{s} \simeq \frac{g_{s}}{\sqrt{4 \pi \mathcal{V}}} M_{\mathrm{Pl}}
$$

with $g_{s}$ being the string coupling. The similar discussion is applied to the case of axiondilaton $\tau$. By contrast, for the internal cycle irrelevant to the visible sector, tiny axion decay constant is achieved in the large volume limit, in particular, phenomenologically favorable axion window, $10^{9} \leq f \leq 10^{12} \mathrm{GeV}$ corresponds to the range, $2 \times 10^{7} \leq \mathcal{V} \leq 6.4 \times 10^{11}$. Furthermore, when we consider the hidden D7-brane wrapping the small cycle in "SwissCheese" CY manifold, the corresponding axion decay constant can be taken much smaller than the string scale independently of the overall volume of CY manifold. In both cases, the string scale is simultaneously small compared with the Planck scale. On the other hand, the trans-Planckian axion decay constant requires the small volume of CY manifold. When the CY volume is of order unity in string units, the quantum corrections will give an important role of determining the axion decay constant as suggested in ref. [18].

In the following, let us take a closer look at the complex structure moduli in type IIB string theory. In contrast to the Kähler moduli in type IIB string, the vacuum expectation values of complex structure moduli are irrelevant to the value of string scale as can be seen in eq. (2.5). It is thus expected that the decay constant of axion associated with complex structure moduli is decoupled from the string scale. Furthermore, since the geometrical and non-perturbative corrections for the complex structure moduli are exactly calculated in the topological string theory, we can estimate such corrections to the axion decay constant around several points of moduli space in a systematic way. Note that, in type IIB (IIA) string theory on toroidal background, axions associated with complex structure (Kähler) moduli couple to the gauge bosons at the one-loop level through the gauge threshold corrections $[28,29]$, although such gauge threshold corrections are unrevealed for the CY background. These one-loop corrections are induced by integrating out the massive open strings between two parallel D-branes. Thus, in a way similar to the toroidal background [28, 29], we assume that the one-loop gauge kinetic function involves the axion associated with complex structure moduli so that the gauge couplings do not diverge around the special points.

Before going to the detail of such corrections in the moduli Kähler potential, we briefly review the Kähler potential written in the language of $\mathcal{N}=2$ special geometry. In type II string theory on CY manifold, the Kähler potential originating from $\mathcal{N}=2$ vector multiplets is provided by the prepotential, which receives the quantum and geometric corrections, calculated by the technique of mirror symmetry between A- and B-models [30, 31]. For sake of simplicity, we consider the type IIB string theory on CY orientifold. On the integral symplectic cohomology basis for $H^{3}(\mathcal{M}, \mathbb{Z})$ of CY manifold, $\left(\alpha_{a}, \beta^{a}\right)$ with $a=1,2, \cdots, h^{2,1}+1$, where $h^{2,1}$ denote the hodge number surviving under the orientifold involution, the holomorphic three-form is represented by

$$
\Omega=z^{a} \alpha_{a}-\mathcal{F}_{a} \beta^{a},
$$


where $z^{a}$ and $\mathcal{F}_{a}=\partial \mathcal{F} / \partial z^{a}$ are defined by the integrals of $\Omega$ over three-cycles $A_{a}$ and $B^{a}$ on the integral homology basis in $H_{3}(\mathcal{M}, \mathbb{Z})$,

$$
z^{a}=\int_{A_{a}} \Omega, \quad \mathcal{F}_{a}=\int_{B^{a}} \Omega,
$$

respectively. Then, $\mathcal{N}=2$ special geometry allows us to write down the Kähler potential of complex structure moduli,

$$
K=-\ln \left[i \int_{\mathcal{M}} \Omega \wedge \bar{\Omega}\right]=-\ln \left[i \Pi^{\dagger} \cdot \Sigma \cdot \Pi\right]
$$

where

$$
\Pi^{t}=\left(\int_{A_{a}} \Omega, \int_{B^{a}} \Omega\right)
$$

is the period vector and $\Sigma$ is the symplectic matrix:

$$
\Sigma=\left(\begin{array}{cc}
0 & 1 \\
-1 & 0
\end{array}\right),
$$

with 1 being the $\left(h^{2,1}+1\right) \times\left(h^{2,1}+1\right)$ identity matrix. When the three-form fluxes are inserted in these three-cycles, the flux-induced superpotential is generated as [32]

$$
W=\int_{\mathcal{M}} G_{3} \wedge \Omega
$$

where $G_{3}=F_{3}-\tau H_{3}$ denote the linear combination of Ramond-Ramond $F_{3}$ and NeveuSchwarz three-form fluxes $H_{3}$. These three-form fluxes enable us to stabilize the complex structure moduli and axion-dilaton [33]. It is remarkable that the Kähler potential is invariant under the symplectic transformation:

$$
\Pi^{i}(z) \rightarrow \sum_{j} P_{j}^{i} \Pi^{j}(z)
$$

where the integral symplectic transformation matrix $P_{j}^{i},\left(i, j=1,2, \cdots, 2\left(h^{2,1}+1\right)\right)$, is the matrix representation of symplectic group $S p\left(2\left(h^{2,1}+1\right)\right)$.

In the complex structure moduli space, there are several special points called as SCS point, conifold point, and LCS point, around which the period vector transforms under the discrete subgroup (monodromy group) of symplectic group,

$$
\sum_{j} P_{j}^{i}\left[z_{\mathrm{sp}}\right] \Pi^{j}(z)=\Pi^{i}\left(z e^{2 \pi i}\right)
$$

with $P_{j}^{i}\left[z_{\mathrm{sp}}\right]$ being the monodromy matrix around the special point $z_{\mathrm{sp}}$. Such symmetries of the Kähler potential around the special points give rise to the flat direction in moduli space. Thus, an axion or axion-like particle naturally appears in the low-energy effective theory.

To estimate the axion decay constant around the special points, it is required to derive an exact form of period vector and its monodromy transformation. In the remaining of this paper, we show the systematic approach to find the general expression of period vector and its monodromy transformation for one-parameter CY manifold within the framework of topological string theory, in which these period vectors are governed by the PicardFuchs differential equations. It thus allows us to study the axion decay constant around the special points of moduli space. 


\section{Axion decay constant around the special point}

We discuss the decay constant of axion around the special points of the complex structure moduli space step by step. First of all, we take into account the SCS points for several CY manifolds in section 3.1. ${ }^{4}$ In section 3.2, we discuss the conifold point of CY manifold, in particular, mirror quintic CY threefold. Finally, the LCS (or large volume) point is discussed in section 3.3.

\subsection{SCS point}

To make the analysis concrete, we, in this section, restrict ourselves to the type IIB string theory on mirror CY orientifold with one complex structure modulus. Furthermore, we concentrate on the complex structure modulus compatible with the orientifold involution.(See for the construction of four-dimensional $\mathcal{N}=1$ effective theory on $\mathrm{CY}$ orientifold, e.g., ref. [27].) In particular, when a CY threefold is defined as a hypersurface in a weighted projective space $\mathbb{P}_{\nu_{1}, \nu_{2}, \cdots, \nu_{n+1}}^{n}\left[d_{1}, d_{2}, \cdots, d_{r}\right]$, where the degree of homogeneous polynomials $d_{1}, d_{2}, \cdots, d_{r}$ satisfies the so-called CY condition: $\sum_{p=1}^{r} d_{p}=\sum_{q=1}^{n+1} \nu_{q}$ with $n=r+3$, the period vector obeys the Picard-Fuchs equation [34, 35]

$$
\left\{\delta^{4}-h z\left(\delta+\alpha_{1}\right)\left(\delta+\alpha_{2}\right)\left(\delta+\alpha_{3}\right)\left(\delta+\alpha_{4}\right)\right\} \pi(z)=0,
$$

where $\delta=z d / d z$ with $z$ being the complex structure modulus, $h=\Pi_{p} d_{p}^{d_{p}} / \Pi_{q} \nu_{q}^{\nu_{q}}$ and $\cup_{i=1}^{4}\left\{\alpha_{i}\right\}=\left\{\cup_{p=1}^{r}\left\{\frac{l}{d_{p}}\right\} \backslash \cup_{q=1}^{n+1}\left\{\frac{m}{\nu_{q}}\right\} \mid 0 \leq l \leq d_{p}-1,0 \leq m \leq \nu_{q}-1\right\}$ for $l, m \in \mathbb{Z}$. The rational numbers $\alpha_{i}$ characterize the CY manifold. Throughout this section, we study the CY manifolds with one complex structure modulus defined in complete intersections in weighted projective spaces and toric varieties. Such CY manifolds are summarized in table 1 in which those are classified by the degeneracies of $\alpha_{i}$. In section 3.1.1, we discuss the case where all $\alpha_{i}$ are distinct, i.e., $\alpha_{1} \neq \alpha_{2} \neq \alpha_{3} \neq \alpha_{4}$, while the other degenerated cases are shown in sections 3.1.2, 3.1.3 and 3.1.4. ${ }^{5}$

The Picard-Fuchs equation involves the regular singular points, i.e., special points, such as the small complex structure point $z=\infty$, conifold point $z=1 / h$ and large complex structure point $z=0$. By solving the above Picard-Fuchs equation around the small complex structure point of CY manifold $z \rightarrow \infty$, the monodromy group is mainly categorized into two classes. Firstly, the monodromy matrix is of finite order, i.e., $P\left[z_{\mathrm{sp}}\right]^{n}=$ $P\left[z_{\mathrm{sp}}\right]$, where $n$ is the integer depending on the structure of CY manifold. In such a case, all $\alpha_{k}$ are distinct and we will call such a special point as orbifold point as discussed in detail in section 3.1.1. Secondly, the monodromy matrix does not obey $P\left[z_{\mathrm{sp}}\right]^{n}=P\left[z_{\mathrm{sp}}\right]$ $(n \in \mathbb{Z})$, while it satisfies $\left(P\left[z_{\mathrm{sp}}\right]^{n}-1\right)^{m}=0$ for certain integers $n$ and $m$. In such a case, some (all) of $\alpha_{k}$ are degenerate and the period vector has a logarithmic behavior as displayed in sections 3.1.2, 3.1.3 and 3.1.4.

First of all, we show the general expression of period vector for the CY manifold with degeneracies of $\alpha_{i}$ less than 2. The solution of Picard-Fuchs equation can be written in

\footnotetext{
${ }^{4}$ In what follows, the mirror CY manifold is denoted by the CY manifold for simplicity, unless otherwise specified.

${ }^{5}$ As pointed out in ref. [36], the case with $\alpha_{1}=\alpha_{2}=\alpha_{3} \neq \alpha_{4}$ will not be realized in toric varieties.
} 


\begin{tabular}{|c|c|c|c|c|c|}
\hline CY hypersurfaces & $\left(\alpha_{1}, \alpha_{2}, \alpha_{3}, \alpha_{4}\right)$ & $h$ & $\kappa$ & $\int_{\mathcal{M}} c_{2} \wedge D$ & Degeneracies of $\alpha_{i}$ \\
\hline $\mathbb{P}_{1,1,1,1,1}^{4}[5]$ & $(1 / 5,2 / 5,3 / 5,4 / 5)$ & 3125 & 5 & 50 & 0 \\
$\mathbb{P}_{2,1,1,1,1}^{4}[6]$ & $(1 / 6,2 / 6,4 / 6,5 / 6)$ & 11664 & 3 & 42 & 0 \\
$\mathbb{P}_{4,1,1,1,1}^{4}[8]$ & $(1 / 8,3 / 8,5 / 8,7 / 8)$ & 65536 & 2 & 44 & 0 \\
$\mathbb{P}_{5,2,1,1,1}^{4}[10]$ & $(1 / 10,3 / 10,7 / 10,9 / 10)$ & $8 \cdot 10^{5}$ & 1 & 34 & 0 \\
$\mathbb{P}_{6,4,1,1,1,1}^{5}[2,12]$ & $(1 / 12,5 / 12,7 / 12,11 / 12)$ & $12^{6}$ & 1 & 46 & 0 \\
$\mathbb{P}_{3,2,2,1,1,1}^{5}[4,6]$ & $(1 / 4,1 / 3,2 / 3,3 / 4)$ & 27648 & 2 & 32 & 0 \\
$\mathbb{P}_{2,1,1,1,1,1}^{5}[3,4]$ & $(1 / 6,1 / 4,3 / 4,5 / 6)$ & 1728 & 6 & 48 & 0 \\
\hline $\mathbb{P}_{1,1,1,1,1,1}^{5}[2,4]$ & $(1 / 4,3 / 4,1 / 2,1 / 2)$ & 1024 & 8 & 56 & 1 \\
$\mathbb{P}_{1,1,1,1,1,1,1}^{6}[2,2,3]$ & $(1 / 3,2 / 3,1 / 2,1 / 2)$ & 432 & 12 & 60 & 1 \\
$\mathbb{P}_{3,1,1,1,1,1}^{5}[2,6]$ & $(1 / 6,5 / 6,1 / 2,1 / 2)$ & 6912 & 4 & 52 & 1 \\
\hline $\mathbb{P}_{1,1,1,1,1,1}^{5}[3,3]$ & $(1 / 3,1 / 3,2 / 3,2 / 3)$ & 729 & 9 & 54 & 2 \\
$\mathbb{P}_{2,2,1,1,1,1}^{5}[4,4]$ & $(1 / 4,1 / 4,3 / 4,3 / 4)$ & 4096 & 4 & 40 & 2 \\
$\mathbb{P}_{3,3,2,2,1,1}^{5}[6,6]$ & $(1 / 6,1 / 6,5 / 6,5 / 6)$ & $2^{8} \cdot 3^{6}$ & 1 & 22 & 2 \\
\hline $\mathbb{P}_{1,1,1,1,1,1,1,1}^{7}[2,2,2,2]$ & $(1 / 2,1 / 2,1 / 2,1 / 2)$ & 256 & 16 & 64 & 4 \\
\hline
\end{tabular}

Table 1. The list of the CY threefolds defined in the ambient spaces $[37],{ }^{6}$ which is classified by the degeneracies of $\alpha_{i}$ with $i=1,2,3,4 . h, \kappa, c_{2}$ and $D$ denote the constant, triple intersection number, the second Chern class of the tangent bundle, and a divisor on the integral basis of CY manifold, respectively. The derivation of $\alpha_{k}$ is shown in ref. [35] for the case of weighted complete intersection.

terms of the integral representation of Meijer $G$-function [36],(For details about the Meijer $G$-function, see, e.g., refs. [36, 38].)

$$
\pi(z)=\left(\begin{array}{c}
U_{0}(z) \\
U_{1}(z) \\
U_{2}(z) \\
U_{3}(z)
\end{array}\right)
$$

where

$$
U_{j}(z)=\frac{1}{(2 \pi i)^{j+1}} \int_{C} d s \frac{\Gamma(-s)^{j+1} \Pi_{i=1}^{4} \Gamma\left(s+\alpha_{i}\right)}{\Gamma(s+1)^{3-j} \Pi_{i=1}^{4} \Gamma\left(\alpha_{i}\right)}\left(e^{\pi i(j+1)} h z\right)^{s},
$$

with $j=0,1,2,3$. In the SCS point, the contour $C$ is taken to extend from $-i \infty$ to $i \infty$ so as to enclose the poles $s=-\alpha_{i}-n$ with $n$ being non-negative integer.

Let us calculate the period integral involving the $n_{s}$ single poles and $n_{d}$ double poles. Although we do not consider the case of triple poles throughout this paper, quadruple pole is discussed in section 3.1.4. The general expression consists of two parts:

$$
U_{j}(z)=U_{j}(z)^{(s)}+U_{j}(z)^{(d)},
$$

\footnotetext{
${ }^{6}$ In ref. [37], there is a typo in the CY data. Specifically, the CY data of $\mathbb{P}_{3,2,2,1,1,1}^{5}[4,6]$ needs to be replaced by that of $\mathbb{P}_{2,1,1,1,1,1}^{5}[3,4]$.
} 
where $U_{j}(z)^{(s)}$ is the contribution from the single poles at $s=-\alpha_{k}-n$

$$
\begin{aligned}
U_{j}(z)^{(s)}= & \frac{1}{(2 \pi i)^{j}} \sum_{k}^{n_{s}}\left(\frac{\sin \left(\pi \alpha_{k}\right)}{\pi}\right)^{3-j}\left(e^{\pi i(j+1)} h z\right)^{-\alpha_{k}} \frac{\Gamma\left(\alpha_{k}\right)^{4} \Pi_{i=1, i \neq k}^{4} \Gamma\left(\alpha_{i}-\alpha_{k}\right)}{\Gamma\left(\alpha_{1}\right) \Gamma\left(\alpha_{2}\right) \Gamma\left(\alpha_{3}\right) \Gamma\left(\alpha_{4}\right)} \\
& \cdot \sum_{n=0}^{\infty} \frac{\left[\left(\alpha_{k}\right)_{n}\right]^{4}}{n ! \Pi_{l=1, \cdots, 4, l \neq k}\left(1+\alpha_{k}-\alpha_{l}\right)_{n}}(h z)^{-n},
\end{aligned}
$$

with $\left(\alpha_{k}\right)_{n}=\frac{\Gamma\left(\alpha_{k}+n\right)}{\Gamma\left(\alpha_{k}\right)}$ being the Pochhammer symbol and the second part $U_{j}(z)^{(d)}$ is originating from the double poles at $s=-\alpha_{k}-n$ depending on the logarithmic term

$$
\begin{aligned}
U_{j}(z)^{(d)}= & \frac{1}{(2 \pi i)^{j}} \sum_{k}^{n_{d}}\left(\frac{\sin \left(\pi \alpha_{k}\right)}{\pi}\right)^{3-j}\left(e^{\pi i(j+1)} h z\right)^{-\alpha_{k}} \\
& \cdot \sum_{n=0}^{\infty} \frac{\Gamma\left(\alpha_{k}+n\right)^{4} \Pi_{i=1, i \neq k, \alpha_{i} \neq \alpha_{k}}^{4} \Gamma\left(\alpha_{i}-\alpha_{k}-n\right)}{n !^{2} \Gamma\left(\alpha_{1}\right) \Gamma\left(\alpha_{2}\right) \Gamma\left(\alpha_{3}\right) \Gamma\left(\alpha_{4}\right)} \\
& \cdot\left[B_{k}^{n}-(3-j) \pi \cot \left(\pi\left(\alpha_{k}+n\right)\right)+\ln \left(e^{\pi i(j+1)} h z\right)\right](h z)^{-n},
\end{aligned}
$$

with $B_{k}^{n}=\sum_{i=1, i \neq k, \alpha_{i} \neq \alpha_{k}}^{4} \Psi\left(\alpha_{i}-\alpha_{k}-n\right)-4 \Psi\left(\alpha_{k}+n\right)-2 \gamma+2 \sum_{l=1}^{n} \frac{1}{l}$. Here, the sum in $U_{j}(z)^{(s)}\left(U_{j}(z)^{(d)}\right)$ is running over the relevant single (double) poles $\alpha_{k}$ and the number of such poles $n_{s}\left(n_{d}\right)$ depends on that of degeneracies of $\alpha_{k} . \Psi(z)=\partial_{z} \ln \Gamma(z)$ is the digamma function which satisfies the identity $\Psi\left(1-\alpha_{k}\right)-\Psi\left(\alpha_{k}\right)=\pi \cot \left(\pi \alpha_{k}\right)$ and $\gamma=-\Psi(1)$ is the Euler-Mascheroni constant. For the degenerate $\alpha_{k}$ in the Picard-Fuchs equation (3.1), the period vectors $U_{j}(z)^{(d)}$ have logarithmic terms depending on the complex structure modulus which causes the infinite order monodromy transformation for the period vector as explained below.

The obtained period vector is not spanned by the integral symplectic basis which is convenient in flux compactification to quantize the three-form fluxes. Therefore, along with the strategy of ref. [24], we search for the transformation matrix from the basis of Meijer $G$-function to the integral symplectic basis. Since the period vector around the LCS point is proportional to the masses of BPS saturated $D_{2 p}$-branes with $p=0,1,2,3$ corresponding to those of three-brane in the mirror IIB string theory [39], we can find the integral basis such that the spectrum between these D-branes are integral. The authors of ref. [24] showed that the integral sympletic basis in the large volume limit $z \rightarrow 0$ becomes

$$
\Pi^{\mathrm{LCS}}=\Xi \cdot \pi(z),
$$

where $U_{j}(z)$ is the element of period vector (3.3) around the LCS point ${ }^{7}$

$$
\Xi=\left(\begin{array}{cccc}
1 & 0 & 0 & 0 \\
-1 & -1 & 0 & 0 \\
-\left(\frac{\kappa b}{4}+\frac{\kappa}{6}\right) & -\left(\frac{\kappa b}{4}+\frac{7 \kappa}{6}\right) & -2 \kappa & -\kappa \\
-\kappa & -2 \kappa & -\kappa & 0
\end{array}\right),
$$

with $b=\frac{1}{3 \kappa} \int_{\mathcal{M}} c_{2} \wedge D$.

\footnotetext{
${ }^{7}$ The contour $C$ is chosen to extend from $-i \infty$ to $i \infty$ so as to enclose the poles $s=n$.
} 
In the following, we assume that the above transformation matrix $\Xi$ is applicable for the period vector around the SCS point $z \rightarrow \infty$ which is confirmed by considering the monodromy transformation of period vector in our discussed concrete models. We find the general form of the period vector on the integral symplectic basis near the SCS point for the case with degeneracies of $\alpha_{i}$ less than 2 ,

$$
\Pi^{\mathrm{SCS}}=\Pi^{s}+\Pi^{d}
$$

where

$$
\begin{aligned}
& \Pi^{s}=\sum_{k}^{n_{s}} \frac{A_{k}}{\pi^{3}}(h z)^{-\alpha_{k}}\left(\begin{array}{c}
s_{k}^{3} e^{-\pi i \alpha_{k}} \\
\frac{i}{2} s_{k}^{2} \\
i \frac{\kappa}{8}\left(b s_{k}^{2}-\left(\frac{1}{3} s_{k}^{2}+c_{k}^{2}\right)\right) \\
\frac{s_{k} \kappa}{4} e^{\pi i \alpha_{k}}
\end{array}\right) \\
& \Pi^{d}=\sum_{k}^{n_{d}} \frac{A_{k}}{\pi^{3}}(h z)^{-\alpha_{k}}\left(\begin{array}{c}
\left.s_{k}^{0}+\ln h+\ln z-3 \pi \frac{c_{k}}{s_{k}}+i \pi\right) e^{-\pi i \alpha_{k}} \\
\frac{i}{2} s_{k}^{2}\left(B_{k}^{0}+\ln h+\ln z-2 \pi \frac{c_{k}}{s_{k}}\right) \\
i \frac{\kappa}{8}\left(b s_{k}^{2}-\left(\frac{1}{3} s_{k}^{2}+c_{k}^{2}\right)\right)\left(B_{k}^{0}+\ln h+\ln z-2 \pi \frac{c_{k}}{s_{k}}\right)-\frac{i \pi c_{k} \kappa}{4 s_{k}} \\
\frac{s_{k} \kappa}{4}\left(B_{k}^{0}+\ln h+\ln z-\pi \frac{c_{k}}{s_{k}}-i \pi\right) e^{\pi i \alpha_{k}}
\end{array}\right),
\end{aligned}
$$

with $s_{k}=\sin \left(\pi \alpha_{k}\right), c_{k}=\cos \left(\pi \alpha_{k}\right)$ and $A_{k}=\frac{\Gamma\left(\alpha_{k}\right)^{4} \Pi_{i=1, i \neq k, \alpha_{i} \neq \alpha_{k}}^{4} \Gamma\left(\alpha_{i}-\alpha_{k}\right)}{\Gamma\left(\alpha_{1}\right) \Gamma\left(\alpha_{2}\right) \Gamma\left(\alpha_{3}\right) \Gamma\left(\alpha_{4}\right)}$. Here, $\Pi^{s}$ $\left(\Pi^{d}\right)$ encodes the contribution from the single (double) poles in the integral of Meijer $G$-function (3.3).

\subsection{1 $\alpha_{1} \neq \alpha_{2} \neq \alpha_{3} \neq \alpha_{4}$}

First of all, we take a closer look at the orbifold point where all $\alpha_{i}$ are distinct. From the formula in eq. (3.10) with $n_{s}=4$ and $n_{d}=0$, the explicit form of period vector becomes

$$
\Pi^{\mathrm{SCS}}=\sum_{k=1}^{4} \Pi^{s, k}=\sum_{k=1}^{4} \frac{A_{k}}{\pi^{3}}(h z)^{-\alpha_{k}}\left(\begin{array}{c}
s_{k}^{3} e^{-\pi i \alpha_{k}} \\
\frac{i}{2} s_{k}^{2} \\
i \frac{\kappa}{8}\left(b s_{k}^{2}-\left(\frac{1}{3} s_{k}^{2}+c_{k}^{2}\right)\right) \\
\frac{s \kappa}{4} e^{\pi i \alpha_{k}}
\end{array}\right)
$$

By plugging the above period vector into eq. (2.8), it enables us to analyze the behavior of the Kähler potential around the SCS point

$$
e^{-K_{\mathrm{SCS}}}=\sum_{k=1}^{4} \frac{A_{k}^{2}}{4 \pi^{6}}|h z|^{-2 \alpha_{k}} s_{k}^{3} c_{k} \kappa\left[-b s_{k}^{2}+2\left(\frac{2}{3} s_{k}^{2}+c_{k}^{2}\right)\right],
$$

where the cross terms in the Kähler potential such as

$$
i \Pi^{\dagger s, i} \cdot \Sigma \cdot \Pi^{s, j}=\frac{A_{i} A_{j}}{\pi^{6}}(h z)^{-\alpha_{i}}(h \bar{z})^{-\alpha_{j}} \frac{\kappa}{8}\left[\sin \left(\left(\alpha_{i}+\alpha_{j}\right) \pi\right)+i\left(s_{i}^{2}-s_{j}^{2}\right)\right]\left[\left(s_{i}^{2}+s_{j}^{2}\right)-\left(b+\frac{2}{3}\right) s_{i}^{2} s_{j}^{2}\right]
$$


with $i \neq j$, are absent in our all concrete models in table $1 .^{8}$ It then implies that the axionic shift symmetry under $\arg (z) \rightarrow \arg (z)+$ const. can be seen in the vicinity of $z \sim \infty$, whose property is originating from the invariance under the following monodromy transformation around the SCS point:

$$
\Pi_{i}^{\mathrm{SCS}}\left(z e^{2 \pi i}\right)=\sum_{j=1}^{4} T_{i j} \Pi_{j}^{\mathrm{SCS}}(z)
$$

with

$$
T=e^{-2 \pi i / d_{c}}\left(\begin{array}{cccc}
1 & 0 & 0 & 0 \\
0 & 1 & 0 & 0 \\
0 & 0 & 1 & 0 \\
0 & 0 & 0 & 1
\end{array}\right)
$$

Here, $d_{c}$ is the least common multiple of the degree of homogeneous polynomials corresponding to the defining equation of CY manifold. We thus find that the monodromy matrix is of finite order,

$$
T^{d_{c}}=\mathbf{1}
$$

as mentioned before.

Following this line of thoughts, we can derive the decay constant of closed string axion $\theta=\arg (z)$

$$
\begin{aligned}
K_{\theta \theta} & =K_{z \bar{z}}|z|^{2} \\
& =-\frac{A_{2}^{2} s_{2}^{3} c_{2}\left(-b s_{2}^{2}+2\left(\frac{2}{3} s_{2}^{2}+c_{2}^{2}\right)\right)}{A_{1}^{2} s_{1}^{3} c_{1}\left(-b s_{1}^{2}+2\left(\frac{2}{3} s_{1}^{2}+c_{1}^{2}\right)\right)}\left(\alpha_{1}-\alpha_{2}\right)^{2} h^{2\left(\alpha_{1}-\alpha_{2}\right)-2}|z|^{2\left(\alpha_{1}-\alpha_{2}\right)}+\mathcal{O}\left(|z|^{2\left(\alpha_{1}-\alpha_{3}\right)}\right),
\end{aligned}
$$

which is valid in the vicinity of $z \sim \infty$. It is remarkable that the factors $|z|^{2}$ in the axion Kähler metric appear as a fact that the axion is now defined in the phase direction of $z$. Since, in all cases, the axion Kähler metric vanishes at the SCS point due to the inequalities $\alpha_{1}<\alpha_{2}<\alpha_{3}<\alpha_{4}$, it enables us to obtain the small axion decay constant around the SCS point. As an example, from the numerical values of Kähler metric in table 2, the small axion Kähler metric $\sqrt{2 K_{\theta \theta}} \simeq 10^{12} \mathrm{GeV}$ is achieved under $|z| \simeq 3.7 \times 10^{3}$ for the $\mathrm{CY}$ manifold defined in $\mathbb{P}_{2,1,1,1,1}^{4}[6]$.

\subsection{2 $\alpha_{1} \neq \alpha_{2} \neq \alpha_{3}=\alpha_{4}$}

In contrast to the previous section, we begin with the case where the single pair of $\alpha_{i}$ is degenerated. From the formula in eq. (3.10) with $n_{s}=2$ and $n_{d}=1$, the period vector is explicitly written by

$$
\Pi^{\mathrm{SCS}}=\sum_{k=1}^{2} \Pi^{s, k}+\Pi^{d},
$$

\footnotetext{
${ }^{8}$ Although the part of obtained Kähler potentials is different from those of previous results in refs. [40, 41] up to overall factors, they are caused by the different normalization of complex structure modulus. Thus, the Kähler metrics are consistent with them when the complex structure modulus is redefined as $\alpha \simeq(h z)^{-\alpha_{1}}$ in the notation of ref. [41].
} 


\begin{tabular}{|c|c|c|}
\hline CY hypersurfaces & $\left(\alpha_{1}, \alpha_{2}, \alpha_{3}, \alpha_{4}\right)$ & $K_{\theta \theta}$ \\
\hline $\mathbb{P}_{1,1,1,1,1}^{4}[5]$ & $(1 / 5,2 / 5,3 / 5,4 / 5)$ & $3.1 \times 10^{-11}|z|^{-2 / 5}$ \\
$\mathbb{P}_{2,1,1,1,1}^{4}[6]$ & $(1 / 6,2 / 6,4 / 6,5 / 6)$ & $1.4 \times 10^{-12}|z|^{-1 / 3}$ \\
$\mathbb{P}_{4,1,1,1,1}^{4}[8]$ & $(1 / 8,3 / 8,5 / 8,7 / 8)$ & $3 \times 10^{-15}|z|^{-1 / 2}$ \\
$\mathbb{P}_{5,2,1,1,1}^{4}[10]$ & $(1 / 10,3 / 10,7 / 10,9 / 10)$ & $1.2 \times 10^{-17}|z|^{-2 / 5}$ \\
$\mathbb{P}_{6,4,1,1,1,1}^{5}[2,12]$ & $(1 / 12,5 / 12,7 / 12,11 / 12)$ & $1.6 \times 10^{-22}|z|^{-2 / 3}$ \\
$\mathbb{P}_{3,2,2,1,1,1}^{5}[4,6]$ & $(1 / 4,1 / 3,2 / 3,3 / 4)$ & $3.2 \times 10^{-10}|z|^{-1 / 6}$ \\
$\mathbb{P}_{2,1,1,1,1,1}^{5}[3,4]$ & $(1 / 6,1 / 4,3 / 4,5 / 6)$ & $5.1 \times 10^{-13}|z|^{-1 / 6}$ \\
\hline
\end{tabular}

Table 2. The axion Kähler metric for the several CY threefolds, where all $\alpha_{i}$ are distinct.

where

$$
\begin{gathered}
\Pi^{s, k}=\frac{A_{k}}{\pi^{3}}(h z)^{-\alpha_{k}}\left(\begin{array}{c}
s_{k}^{3} e^{-\pi i \alpha_{k}} \\
\frac{i}{2} s_{k}^{2} \\
i \frac{\kappa}{8}\left(b s_{k}^{2}-\left(\frac{1}{3} s_{k}^{2}+c_{k}^{2}\right)\right) \\
\frac{s_{k} \kappa}{4} e^{\pi i \alpha_{k}}
\end{array}\right) \\
\Pi_{3}^{d}=\frac{A_{3}}{\pi^{3}}(h z)^{-\alpha_{3}}\left(\begin{array}{c}
\left.B_{3}^{0}+\ln h+\ln z-3 \pi \frac{c_{3}}{s_{3}}+i \pi\right) e^{-\pi i \alpha_{3}} \\
\frac{i}{2} s_{3}^{2}\left(B_{3}^{0}+\ln h+\ln z-2 \pi \frac{c_{3}}{s_{3}}\right) \\
i \frac{\kappa}{8}\left(b s_{3}^{2}-\left(\frac{1}{3} s_{3}^{2}+c_{3}^{2}\right)\right)\left(B_{3}^{0}+\ln h+\ln z-2 \pi \frac{c_{3}}{s_{3}}\right)-\frac{i \pi c_{3} \kappa}{4 s_{3}} \\
\frac{s_{3} \kappa}{4}\left(B_{3}^{0}+\ln h+\ln z-\pi \frac{c_{3}}{s_{3}}-i \pi\right) e^{\pi i \alpha_{3}}
\end{array}\right) .
\end{gathered}
$$

The above period vector leads to the Kähler potential for each CY manifold by the use of eq. (2.8),

$$
\begin{aligned}
e^{-K_{\mathrm{SCS}}}= & \sum_{k=1}^{2} \frac{A_{k}^{2}}{4 \pi^{6}}|h z|^{-2 \alpha_{k}} s_{k}^{3} c_{k} \kappa\left[-b s_{k}^{2}+2\left(\frac{2}{3} s_{k}^{2}+c_{k}^{2}\right)\right] \\
& -\frac{\kappa A_{3}^{2}}{8 \pi^{5}}|h z|^{-2 \alpha_{3}}\left(b-\frac{4}{3}\right)\left(2 B_{3}+\ln |h z|^{2}\right),
\end{aligned}
$$

around the SCS point. Here, the cross terms such as $i\left(\Pi^{\dagger s}\right)^{\dagger} \cdot \Sigma \cdot \Pi^{d}$ are absent, since they are proportional to the following equalities satisfied in our concrete models in table 1 ,

$$
s_{k}^{2}\left(b-\frac{1}{3}\right)-1=0
$$

with $k=1,2$. Thus, the obtained Kähler potential has the axionic shift symmetry under $\theta \rightarrow \theta+$ const. with $\theta=\arg (z)$ which is originating from the following fact. Around the SCS point, the period vector transforms as

$$
\Pi^{\mathrm{SCS}}\left(z e^{2 \pi i}\right)=\sum_{k=1}^{2} e^{-2 \pi i \alpha_{k}} \Pi^{s, k}+e^{-2 \pi i \alpha_{3}} \Pi^{d}+2 \pi i \xi^{\mathrm{SCS}}
$$




\begin{tabular}{|c|c|c|}
\hline CY hypersurfaces & $\left(\alpha_{1}, \alpha_{2}, \alpha_{3}, \alpha_{4}\right)$ & $K_{\theta \theta}$ \\
\hline $\mathbb{P}_{1,1,1,1,1,1}^{5}[2,4]$ & $(1 / 4,3 / 4,1 / 2,1 / 2)$ & $9.3 \times 10^{-5}|z|^{-1 / 2} \ln |z|$ \\
$\mathbb{P}_{1,1,1,1,1,1,1}^{6}[2,2,3]$ & $(1 / 3,2 / 3,1 / 2,1 / 2)$ & $3.6 \times 10^{-4}|z|^{-1 / 3} \ln |z|$ \\
$\mathbb{P}_{3,1,1,1,1,1}^{5}[2,6]$ & $(1 / 6,5 / 6,1 / 2,1 / 2)$ & $4.7 \times 10^{-6}|z|^{-2 / 3} \ln |z|$ \\
\hline
\end{tabular}

Table 3. The axion Kähler metric for the several CY threefolds, where one pair of $\alpha_{i}$ is degenerated.

where

$$
\xi^{\mathrm{SCS}}=\frac{A_{3}}{\pi^{3}}(h z)^{-\alpha_{3}}\left(\begin{array}{c}
s_{3}^{3} e^{-\pi i \alpha_{3}} \\
\frac{i}{2} s_{3}^{2} \\
i \frac{\kappa}{8}\left(b s_{3}^{2}-\left(\frac{1}{3} s_{3}^{2}+c_{3}^{2}\right)\right) \\
\frac{s_{k} \kappa}{4} e^{\pi i \alpha_{3}}
\end{array}\right)
$$

In turn, we find the monodromy matirix $T$ obeying ${ }^{9}$

$$
\Pi_{i}^{\mathrm{SCS}}\left(z e^{2 \pi i}\right)=\sum_{j=1}^{4} T_{i j} \Pi_{j}^{\mathrm{SCS}}(z),
$$

generically satisfies the following equality, even if we do not know its explicit form ${ }^{10}$

$$
\left(T^{d_{c}}-\mathbf{1}\right)^{2}=0
$$

which corresponds to the symmetry of Kähler potential for each CY manifold. The above equality is originating from the fact that the operator $-\left(T^{d_{c}}\right)^{2}+2 T^{d_{c}}$ is identified with the identity operator, i.e., 1.

The Kähler metric of axion is then obtained as

$$
\begin{aligned}
K_{\theta \theta} & =K_{z \bar{z}}|z|^{2} \\
& \simeq \frac{\pi A_{3}^{2}\left(b-\frac{4}{3}\right)}{2 A_{1}^{2} s_{1}^{3} c_{1}\left(-b s_{1}^{2}+2\left(\frac{2}{3} s_{1}^{2}+c_{1}^{2}\right)\right)}\left[\left(\alpha_{1}-\alpha_{3}\right)^{2}\left(2 B_{3}^{0}+\ln |h z|^{2}\right)+\alpha_{1}-\alpha_{3}+1\right]|h z|^{2\left(\alpha_{1}-\alpha_{3}\right)},
\end{aligned}
$$

which is valid in the vicinity of $z \sim \infty$. Since the axion Kähler metric vanishes at the SCS point of CY manifold due to the inequalities $\alpha_{1}<\alpha_{3}=\alpha_{4}<\alpha_{2}$ as shown in table 1, we can obtain the small axion decay constant around the SCS point. In table 3, we show the numerical values of Kähler metric for the CY manifold in table 1. As an example, from the numerical values of Kähler metric in table 3 , the small axion Kähler metric $\sqrt{2 K_{\theta \theta}} \simeq 10^{12} \mathrm{GeV}$ is achieved under $|z| \simeq 7.2 \times 10^{13}$ for the $\mathrm{CY}$ manifold defined in $\mathbb{P}_{3,1,1,1,1,1}^{5}[2,6]$.

\footnotetext{
${ }^{9}$ In ref. [24], the monodromy matrix is also constructed on the Jordan basis of Meijer $G$-function.

${ }^{10}$ The explicit form of monodromy matrix $T$ can be derived as follows. First, we obtain the monodromy matrix of the period vector around the SCS point on the basis of eq. (3.2), known as the Jordan basis [36]. Then, by acting the transformation matrix $\Xi$ in eq. (3.7) on the obtained monodromy matrix of the Jordan basis, the monodromy matirix $T$ is obtained.
} 


\subsection{3 $\alpha_{1}=\alpha_{2} \neq \alpha_{3}=\alpha_{4}$}

Next, we consider the CY manifold with $\alpha_{1}=\alpha_{2} \neq \alpha_{3}=\alpha_{4}$ which causes the double poles around the SCS point.

Following the same procedure in section 3.1.2, the period vector on the integral symplectic basis is obtained from the formula in eq. (3.10) with $n_{s}=0$ and $n_{d}=2$,

$$
\begin{aligned}
\Pi^{\mathrm{SCS}} & =\sum_{k=1,3} \Pi^{d, k} \\
& =\sum_{k=1,3} \frac{A_{k}}{\pi^{3}}(h z)^{-\alpha_{k}}\left(\begin{array}{c}
s_{k}^{3}\left(B_{k}^{0}+\ln h+\ln z-3 \pi \frac{c_{k}}{s_{k}}+i \pi\right) e^{-\pi i \alpha_{k}} \\
\frac{i}{2} s_{k}^{2}\left(B_{k}^{0}+\ln h+\ln z-2 \pi \frac{c_{k}}{s_{k}}\right) \\
i \frac{\kappa}{8}\left(b s_{k}^{2}-\left(\frac{1}{3} s_{k}^{2}+c_{k}^{2}\right)\right)\left(B_{k}^{0}+\ln h+\ln z-2 \pi \frac{c_{k}}{s_{k}}\right)-\frac{i \pi c_{k} \kappa}{4 s_{k}} \\
\frac{s_{k} \kappa}{4}\left(B_{k}^{0}+\ln h+\ln z-\pi \frac{c_{k}}{s_{k}}-i \pi\right) e^{\pi i \alpha_{k}}
\end{array}\right),
\end{aligned}
$$

which leads to the Kähler potential

$$
e^{-K_{\mathrm{SCS}}}=\sum_{k=1,3} \frac{\kappa A_{k}^{2}}{2 \pi^{5}}|h z|^{-2 \alpha_{k}} s_{k}^{2} c_{k}^{2}\left[\ln |h z|^{2}+2 B_{k}-6 \pi \frac{c_{k}}{s_{k}}+\frac{\pi}{s_{k} c_{k}}\right] .
$$

Here, the cross term $i \Pi^{\dagger d, 1} \cdot \Sigma \cdot \Pi^{d, 3}$ is absent by the use of following equalities satisfied in our concrete models in table 1 ,

$$
s_{k}^{2}\left(b+\frac{2}{3}\right)-2=0
$$

with $k=1,3$. Thus, the obtained Kähler potential has the axionic shift symmetry under $\theta \rightarrow \theta+$ const. with $\theta=\arg (z)$ which is originating from the following fact.

Around the SCS point, the period vector transforms as

$$
\Pi^{\mathrm{SCS}}\left(z e^{2 \pi i}\right)=\sum_{k=1,3} e^{-2 \pi i \alpha_{k}} \Pi^{d, k}+2 \pi i \sum_{k=1,3} \xi^{s, k},
$$

where

$$
\xi^{s, k}=\frac{A_{k}}{\pi^{3}}(h z)^{-\alpha_{k}}\left(\begin{array}{c}
s_{k}^{3} e^{-\pi i \alpha_{k}} \\
\frac{i}{2} s_{k}^{2} \\
i \frac{\kappa}{8}\left(b s_{k}^{2}-\left(\frac{1}{3} s_{k}^{2}+c_{k}^{2}\right)\right) \\
\frac{s_{k} \kappa}{4} e^{\pi i \alpha_{k}}
\end{array}\right)
$$

In turn, we find the monodromy matirix $T$ obeying

$$
\Pi_{i}^{\mathrm{SCS}}\left(z e^{2 \pi i}\right)=\sum_{j=1}^{4} T_{i j} \Pi_{j}^{\mathrm{SCS}}(z),
$$

generically satisfies the following equality, even if we do not know its explicit form,

$$
\left(T^{d_{c}}-\mathbf{1}\right)^{2}=0
$$


which corresponds to the symmetry of Kähler potential for each CY manifold in the same way with section 3.1.2. The Kähler metric of axion is then obtained in the vicinity of $z \sim \infty$,

$$
\begin{aligned}
K_{\theta \theta}= & K_{z \bar{z}}|z|^{2} \\
\simeq & \left(\alpha_{1}\right)^{2}\left(\frac{\left[\ln |h z|^{2}+2 B_{1}-6 \pi \frac{c_{1}}{s_{1}}+\frac{\pi}{s_{1} c_{1}}-\frac{1}{\alpha_{1}}\right]^{2}}{\left[\ln |h z|^{2}+2 B_{1}-6 \pi \frac{c_{1}}{s_{1}}+\frac{\pi}{s_{1} c_{1}}\right]^{2}}-\frac{\ln |h z|^{2}+2 B_{1}-6 \pi \frac{c_{1}}{s_{1}}+\frac{\pi}{s_{1} c_{1}}-\frac{2}{\alpha_{1}}}{\ln |h z|^{2}+2 B_{1}-6 \pi \frac{c_{1}}{s_{1}}+\frac{\pi}{s_{1} c_{1}}}\right) \\
& +\mathcal{O}\left(|z|^{2\left(\alpha_{1}-\alpha_{3}\right)} \ln |z|^{2}\right),
\end{aligned}
$$

from which the axion Kähler metric vanishes at the SCS point of CY manifold by using the inequalities $\alpha_{1}=\alpha_{2}<\alpha_{3}=\alpha_{4}$. In contrast to the previous case, constrained axion Kähler metric $\sqrt{2 K_{\theta \theta}} \simeq \mathcal{O}\left(10^{12}\right) \mathrm{GeV}$ requires the value of complex structure modulus $|z|$ is of $\mathcal{O}\left(10^{300}\right)$ due to the logarithmic dependence of the Kähler metric.

\subsection{4 $\alpha_{1}=\alpha_{2}=\alpha_{3}=\alpha_{4}$}

In this section, we consider the CY manifold with $\alpha_{1}=\alpha_{2}=\alpha_{3}=\alpha_{4}$ which causes the quadrupole poles around the SCS point as shown in the Meijer integral (3.3). So far, the SCS point can be distinguishable from the LCS point in the CY manifold. However, in the current case, Picard-Fuchs equation around the SCS point is related to that around the LCS point. Along the line of ref. [42], under the following change of variable $z$ and solution of Picard-Fuchs equation in eq. (3.1):

$$
\begin{aligned}
u & =z^{-1}, \\
\tilde{\pi}(u) & =z^{-\alpha_{1}} \pi(z),
\end{aligned}
$$

we have obtain the same Picard-Fuchs equation (3.1),

$$
\left\{\delta_{u}^{4}-h u\left(\delta_{u}+\alpha_{1}\right)^{4}\right\} \pi(u)=0
$$

where $\delta_{u}=u d / d u=-z d / d z$. It implies that the SCS point is physically equivalent to the LCS point. Indeed, when the contour $C$ in eq. (3.3) is taken to enclose such quadrupole poles, the Meijer $G$-function has the logarithmic terms [42]:

$U_{j}(z)=\frac{1}{(2 \pi i)^{j}}\left(\frac{\sin \pi \alpha}{\pi}\right)^{3-j}\left(e^{\pi i(j+1)} h z\right)^{-\alpha_{1}}\left[(\log (h z))^{3}+D_{2}^{j}(\log (h z))^{2}+D_{1}^{j} \log (h z)+D_{0}^{j}\right]$,

where

$$
\begin{aligned}
D_{0}^{j} & =\frac{32}{3} \gamma^{3}+\frac{4}{3} \gamma \pi^{2}+\frac{4}{3} \xi(2)+\left(8 \gamma^{2}+\frac{1}{3} \pi^{2}\right) C^{j}+4 \gamma\left(C^{j 2}+C^{j^{\prime}}\right)+C^{j 3}+3 C^{j} C^{j^{\prime}}+C^{j^{\prime \prime}}, \\
D_{1}^{j} & =8 \gamma^{2}+\frac{1}{3} \pi^{2}+8 \gamma C^{j}+3\left(C^{j}\right)^{3}+3 C^{j^{\prime}}, \\
D_{2}^{j} & =4 \gamma+3 C^{j},
\end{aligned}
$$


with

$$
\begin{aligned}
C^{j} & \left.\equiv \mathcal{C}^{j}\right|_{s=-\alpha}, \\
C^{j^{\prime}} & \left.\equiv \frac{d}{d s} \mathcal{C}^{j}\right|_{s=-\alpha}, \\
C^{j^{\prime \prime}} & \left.\equiv \frac{d^{2}}{d s^{2}} \mathcal{C}^{j}\right|_{s=-\alpha}, \\
\mathcal{C}^{j} & =\log k+(j+1) i \pi-(j+1) \Psi(-s)-(j-3) \Psi(1+s) .
\end{aligned}
$$

Such cubic term of logarithm induced by the existence of quadrupole poles, also appears in the solution of Picard-Fuchs equation around the LCS point as discussed in section 3.3. Thus, even if the small Kähler metric is obtained around the SCS point, the Kähler metric corresponds to that around the large complex modulus limit. We analyze the detail of such a case in section 3.3 .

\subsection{Conifold point}

In a way similar to the previous sections, we next analyze the decay constant of closed string axion around other special point, in particular, conifold point which often appears in the landscape of string theory [43] and the vicinity of large number of D3-branes [44]. For our purpose, we study the type IIB string theory compactified on the mirror quintic CY threefold which encodes the conifold point. However, the solution of Picard-Fuchs equation around the conifold point cannot be described by the Meijer $G$-function.

To obtain the period vector around the conifold point, we directly solve the PicardFuchs equation around the conifold point. First of all, when we redefine the complex structure modulus as $z_{\mathrm{c}}=1-\psi^{-5}$ in the defining equation of mirror quintic,

$$
\mathcal{P}_{1}=\left(x_{0}\right)^{5}+\left(x_{1}\right)^{5}+\left(x_{2}\right)^{5}+\left(x_{3}\right)^{5}+\left(x_{4}\right)^{5}-5 \psi \Pi_{i=0}^{4} x_{i}=0, \quad\left(x_{i} \in \mathbb{P}_{1,1,1,1,1}^{4}[5]\right),
$$

associated Picard-Fuchs equation in eq. (3.1) reduces to

$$
\left\{P_{4}\left(z_{\mathrm{c}}\right) \theta_{\mathrm{c}}^{4}+P_{3}\left(z_{\mathrm{c}}\right) \theta_{\mathrm{c}}^{3}+P_{2}\left(z_{\mathrm{c}}\right) \theta_{\mathrm{c}}^{2}+P_{1}\left(z_{\mathrm{c}}\right) \theta_{\mathrm{c}}^{1}+P_{0}\left(z_{\mathrm{c}}\right)\right\} \pi^{c}\left(z_{\mathrm{c}}\right)=0,
$$

where $\theta_{\mathrm{c}}=z_{\mathrm{c}} d / d z_{\mathrm{c}}$ and

$$
\begin{aligned}
& P_{4}\left(z_{\mathrm{c}}\right)=1-3 z_{\mathrm{c}}+3 z_{\mathrm{c}}^{2}-z_{\mathrm{c}}^{3}, \\
& P_{3}\left(z_{\mathrm{c}}\right)=-4+6 z_{\mathrm{c}}-14 z_{\mathrm{c}}^{3}, \\
& P_{2}\left(z_{\mathrm{c}}\right)=\frac{1}{5}\left(25-22 z_{\mathrm{c}}+4 z_{\mathrm{c}}^{2}+103 z_{\mathrm{c}}^{3}\right), \\
& P_{1}\left(z_{\mathrm{c}}\right)=\frac{1}{5}\left(-10+7 z_{\mathrm{c}}-2 z_{\mathrm{c}}^{3}\right), \\
& P_{0}\left(z_{\mathrm{c}}\right)=-\frac{24}{5^{5}} z_{\mathrm{c}}^{3} .
\end{aligned}
$$

When we take the following ansatz of solution of Picard-Fuchs equation,

$$
\pi^{\mathrm{c}}\left(z_{\mathrm{c}}\right)=\sum_{n=0}^{\infty} \alpha_{n} z_{\mathrm{c}}^{\rho+n},
$$


the characteristic exponent of Picard-Fuchs operator is obtained as $\rho=0,1,1,2$ which results in the period vector by using the recursive approach,

$$
\pi^{\mathrm{c}}\left(z_{\mathrm{c}}\right)=\left(\begin{array}{l}
\pi_{1}^{\mathrm{c}} \\
\pi_{2}^{\mathrm{c}} \\
\pi_{3}^{\mathrm{c}} \\
\pi_{4}^{\mathrm{c}}
\end{array}\right)=\left(\begin{array}{c}
1+\frac{2}{5^{4}} z_{\mathrm{c}}^{3}+\frac{97}{2 \cdot 4 \cdot 5} z_{\mathrm{c}}^{4}+\cdots \\
z_{\mathrm{c}}+\frac{7}{10} z_{\mathrm{c}}^{2}+\frac{41}{75} z_{\mathrm{c}}^{3}+\frac{1133}{4 \cdot 5^{5}} z_{\mathrm{c}}^{4}+\cdots \\
z_{\mathrm{c}}^{2}+\frac{37}{30} z_{\mathrm{c}}^{3}+\frac{2309}{1800} z_{\mathrm{c}}^{4}+\cdots \\
\pi_{2} \ln z-\frac{23}{360} z_{\mathrm{c}}^{3}-\frac{6397}{3 \cdot 10^{6}} z_{\mathrm{c}}^{4}+\cdots
\end{array}\right),
$$

However, the obtained period vector is not spanned by the integral symplectic basis. By acting the transformation matrix ${ }^{11}$

$$
\Xi^{\mathrm{c}}=\left(\begin{array}{cccc}
a_{4} & a_{5} & a_{9} & -\frac{\sqrt{5}}{(2 \pi i)^{2}} \\
i a_{6} & i a_{7} & i a_{8} & 0 \\
0 & \frac{\sqrt{5}}{2 \pi i} & 0 & 0 \\
a_{1}-\frac{11}{2} i a_{6} & a_{2}-\frac{11}{2} i a_{7} & a_{3}-\frac{11}{2} i a_{8} & 0
\end{array}\right)
$$

with the period vector (3.44), the period vector on the integral symplectic basis can be obtained around the conifold point

$$
\Pi^{\mathrm{c}}=\left(\begin{array}{c}
-\frac{\sqrt{5}}{(2 \pi i)^{2}} z_{\mathrm{c}} \ln z_{\mathrm{c}}+a_{4}+a_{5} z_{\mathrm{c}}+\mathcal{O}\left(z_{\mathrm{c}}^{2}\right) \\
i a_{6}+a_{11} z_{\mathrm{c}}+\mathcal{O}\left(z_{\mathrm{c}}^{2}\right) \\
\frac{\sqrt{5}}{2 \pi i} z_{\mathrm{c}}+\mathcal{O}\left(z_{\mathrm{c}}^{2}\right) \\
a_{12}+a_{10} z_{\mathrm{c}}+\mathcal{O}\left(z_{\mathrm{c}}^{2}\right)
\end{array}\right)
$$

with $a_{10}=1.016604716702582-0.8292168231795108 i$ and $a_{11}=0.15076669512354743 i$, and $a_{12}=a_{1}-7.114656914772605 i$.

From the Kähler potential

$$
e^{-K}=i\left(\Pi^{\mathrm{c}}\right)^{\dagger} \cdot \Sigma \cdot \Pi^{\mathrm{c}}=\frac{5}{(2 \pi)^{3}}\left|z_{\mathrm{c}}\right|^{2} \ln \left|z_{\mathrm{c}}\right|^{2}+C+D\left(z_{\mathrm{c}}+\bar{z}_{\mathrm{c}}\right)+E\left|z_{\mathrm{c}}\right|^{2},
$$

with $C \simeq 16.02, D \simeq 2.63$ and $E \simeq 0.289$, we identify the phase of complex structure modulus as axion $z_{\mathrm{c}}=e^{2 \pi i \theta_{\mathrm{c}}}$, since the Kähler potential has the discrete shift symmetry under $\theta_{\mathrm{c}} \rightarrow \theta_{\mathrm{c}}+N$ with $N$ being integer. Such a symmetry is originating from the following monodromy transformation. Around the conifold point, the period vector transforms as

$$
\Pi^{\mathrm{c}}\left(z e^{2 \pi i}\right)=\Pi^{\mathrm{c}}(z)+\xi^{\mathrm{c}}(z)
$$

where

$$
\xi^{\mathrm{c}}=-\frac{5}{(2 \pi i)^{2}} z_{\mathrm{c}}\left(\begin{array}{l}
1 \\
0 \\
0 \\
0
\end{array}\right)
$$

\footnotetext{
${ }^{11}$ The elements of transformation matrix is only numerically known in ref. [45] as $a_{1}=6.19501627714957$, $a_{2}=1.016604716702582, a_{3}=-0.140889979448831, a_{4}=1.07072586843016, a_{5}=-0.0247076138044847$, $a_{6}=1.29357398450411, a_{7}=\frac{2 a_{2} a_{6} \pi-\sqrt{5} a_{4}}{2 a_{1} \pi}, a_{8}=\frac{5+16 a_{3} a_{6} \pi^{3}}{16 a_{1} \pi^{3}}, a_{9}=\frac{\sqrt{5} a_{2}+8 a_{3} a_{4} \pi^{2}}{8 a_{1} \pi^{2}}$.
} 
Consequently, under the monodromy transformation:

$$
\Pi_{i}^{\mathrm{c}} \rightarrow \sum_{j=1}^{4} T_{i j}^{\mathrm{c}} \Pi_{j}^{\mathrm{c}},
$$

we find that the transformation matrix $T^{\mathrm{c}}$ satisfies the following equalities, even if we do not know its explicit form, ${ }^{12}$

$$
\left(T^{\mathrm{c}}-\mathbf{1}\right)^{2}=0,
$$

which corresponds to the symmetry of Kähler potential in the same way with sections 3.1.2 and 3.1.3.

In the following, we proceed to study the decay constant of axion $\theta_{\mathrm{c}}$. The Kähler metric of axion $\theta_{\mathrm{c}}$ becomes

$$
\begin{aligned}
K_{\theta_{\mathrm{c}} \theta_{\mathrm{c}}} & =K_{z_{\mathrm{c}} \bar{z}_{\mathrm{c}}}\left|z_{\mathrm{c}}\right|^{2} \\
& =\frac{\left|z_{\mathrm{c}}\right|^{2}}{V_{\mathrm{c}}^{2}}\left|\frac{5}{(2 \pi)^{3}}\left(z_{\mathrm{c}} \ln \left|z_{\mathrm{c}}\right|^{2}+z_{\mathrm{c}}\right)+D+E z_{\mathrm{c}}\right|^{2}-\frac{\left|z_{\mathrm{c}}\right|^{2}}{V_{\mathrm{c}}}\left(\frac{5}{(2 \pi)^{3}} \ln \left|z_{\mathrm{c}}\right|^{2}+\frac{10}{(2 \pi)^{3}}+E\right),
\end{aligned}
$$

with $V_{\mathrm{c}}=e^{-K}$, which approaches to zero in the limit of $z_{\mathrm{c}} \rightarrow 0$. We will thus expect that the axion decay constant can be taken much smaller than the string scale. Indeed, from the Kähler metric in eq. (3.52), the small axion Kähler metric $\sqrt{2 K_{\theta_{\mathrm{c}} \theta_{\mathrm{c}}}} \simeq 10^{12} \mathrm{GeV}$ is achieved under $\left|z_{c}\right| \simeq 1.6 \times 10^{-6}$ for the mirror quintic $C Y$ defined in $\mathbb{P}_{1,1,1,1,1}^{4}[5]$.

\subsection{LCS point}

Finally, we discuss the decay constant of single and multiple axions around the LCS point based on the effective action of type IIA string theory on CY manifold $\tilde{\mathcal{M}}$. By applying the mirror map for the period vector of Picard-Fuchs equation around the LCS point, $t^{i} \simeq \frac{\ln z^{i}}{2 \pi i}$ with $i=1,2, \cdots, h^{2,1}$, we can obtain the period vector around the large volume point in the type IIA string theory. The authors of refs. $[34,35]$ showed that the period vector around the large volume point is represented by

$$
\Pi^{\mathrm{LCS}}=\left(\begin{array}{c}
1 \\
t^{i} \\
2 F-t^{i} \partial_{i} F \\
\partial_{i} F
\end{array}\right),
$$

where $F$ is the instanton-corrected prepotential ${ }^{13}$

$$
F=-\frac{1}{3 !} \kappa_{i j k} t^{i} t^{j} t^{k}-\frac{1}{2} \kappa_{i j} t^{i} t^{j}+\kappa_{i} t^{i}+\frac{1}{2} \kappa_{0}-\frac{1}{(2 \pi i)^{3}} \sum_{\beta} n_{\beta} \operatorname{Li}_{3}\left(q^{\beta}\right),
$$

with $\operatorname{Li}_{s}(z)=\sum_{n=1} \frac{z^{n}}{n^{s}}$ being polylogarithm function. Here, $q^{\beta_{i}}=e^{2 \pi i d_{i} t^{i}}$ denote the instanton corrections labeled by instanton number $n_{\beta}$, where $\beta=d_{i} \beta_{i}$ with $d_{i}$ being integers

\footnotetext{
${ }^{12}$ The explicit form of $T^{\mathrm{c}}$ is obtained in a similar way with section 3.1.2.

${ }^{13}$ Here and in what follows, the summation over $i, j, k=1,2, \cdots, h^{2,1}$ is implied.
} 
is expanded on the integral cohomology basis for $H_{2}(\tilde{\mathcal{M}}, \mathbb{Z}) \backslash\{0\}$ of the $\mathrm{CY}$ manifold $\tilde{\mathcal{M}}$. Furthermore, the topological invariant quantities are also defined in terms of the Kähler form $J_{i}$ of $\tilde{\mathcal{M}}$,

$$
\begin{aligned}
\kappa_{i j k} & =\int_{\tilde{\mathcal{M}}} J_{i} \wedge J_{j} \wedge J_{k}, & \kappa_{i j} & =-\frac{1}{2} \int_{\tilde{\mathcal{M}}} J_{i} \wedge J_{j} \wedge J_{j} \\
\kappa_{j} & =\frac{1}{24} \int_{\tilde{\mathcal{M}}} c_{2}(\tilde{\mathcal{M}}) \wedge J_{j}, & \kappa_{0} & =\frac{\zeta(3)}{(2 \pi i)^{3}} \int_{\tilde{\mathcal{M}}} c_{3}(\tilde{\mathcal{M}})=\frac{\zeta(3)}{(2 \pi i)^{3}} \chi(\tilde{\mathcal{M}}),
\end{aligned}
$$

where $\zeta(3) \simeq 1.2$ and $\chi(\tilde{\mathcal{M}})$ is the Euler characteristic.

As shown below, the Kähler potential constructed by the period vector (3.53) involves the axionic shift symmetries $t^{i} \rightarrow t^{i}+1$, originating from the gauge symmetry of KalbRamond field Re $t^{i}$. Indeed, the period vector (3.53) around the LCS point is transformed under $t^{i} \rightarrow t^{i}+1$ for $i=1,2, \cdots, h^{2,1}$,

$$
\Pi^{\mathrm{LCS}}\left(t^{i}+1\right)=T^{\mathrm{LCS}} \cdot \Pi^{\mathrm{LCS}}\left(t^{i}\right)
$$

where

$$
T^{\mathrm{LCS}}=\left(\begin{array}{cc}
\mathcal{T}_{1} & 0 \\
\mathcal{T}_{2} & \mathcal{T}_{3}
\end{array}\right)
$$

with

$$
\begin{aligned}
\mathcal{T}_{1}= & \left(\begin{array}{ccccc}
1 & 0 & 0 & \cdots & 0 \\
1 & 1 & 0 & \cdots & 0 \\
1 & 0 & 1 & \cdots & 0 \\
\vdots & \vdots & \vdots & \ddots & \vdots \\
1 & 0 & 0 & \cdots & 1
\end{array}\right), \quad \mathcal{T}_{2}=\left(\begin{array}{cccc}
\sum_{i} \kappa_{i} & \mathcal{A}_{1} & \cdots & \mathcal{A}_{h^{2,1}} \\
-\mathcal{A}_{1} & -\sum_{j} \kappa_{1 j 1} & \cdots & -\sum_{j} \kappa_{1 j h^{2,1}} \\
\vdots & \vdots & \vdots & \vdots \\
-\mathcal{A}_{h^{2,1}} & -\sum_{j} \kappa_{h^{2,1} j 1} & \cdots & -\sum_{j} \kappa_{h^{2,1} j h^{2,1}}
\end{array}\right) \\
\mathcal{T}_{3} & \left(\begin{array}{ccccc}
1 & -1 & -1 & \cdots & -1 \\
0 & 1 & 0 & \cdots & 0 \\
0 & 0 & 1 & \cdots & 0 \\
\vdots & \vdots & \vdots & \ddots & \vdots \\
0 & 0 & 0 & \cdots & 1
\end{array}\right)
\end{aligned}
$$

and $\mathcal{A}_{i}=\frac{1}{2}\left(\sum_{j, k} \kappa_{i j k}-\sum_{j}\left(\kappa_{i j}+\kappa_{j i}\right)\right)$. The above monodromy matrix $T^{\mathrm{LCS}}$ satisfies the following maximally unipotent property,

$$
\left(T^{\mathrm{LCS}}-\mathbf{1}\right)^{2\left(h^{2,1}+1\right)}=0,
$$

which leads to the symmetries of the Kähler potential as shown below.

In the remaining of this section, we analyze the axion decay constant around the LCS point with single axion in section 3.3.1 and multiple axions in section 3.3.2. 


\subsubsection{Single axion}

We begin with the single axion case. From the period vector in eq. (3.53), the quantumcorrected Kähler potential for single modulus $T=i t$ is represented by

$$
\begin{aligned}
K(T, \bar{T})=-\ln & {\left[\frac{1}{6} \kappa_{t t t}(T+\bar{T})^{3}-\frac{\zeta(3)}{4 \pi^{3}} \chi(\tilde{\mathcal{M}})\right.} \\
& +\frac{2}{(2 \pi)^{2}} \sum_{n, d_{t}=1}^{\infty} \frac{d_{t} n_{\beta}}{n^{2}}(T+\bar{T}) e^{-\pi n d_{t}(T+\bar{T})} \cos \left(-i \pi n d_{t}(T-\bar{T})\right) \\
& \left.+\frac{4}{(2 \pi)^{3}} \sum_{n, d_{t}=1}^{\infty} \frac{n_{\beta}}{n^{3}} e^{-\pi n d_{t}(T+\bar{T})} \cos \left(-i \pi n d_{t}(T-\bar{T})\right)\right]
\end{aligned}
$$

in the large volume limit, where $\zeta(3) \simeq 1.2, \kappa_{t t t}$ denotes the classical intersection number of CY manifold, and the exponential terms show the instanton corrections labeled by the instanton number $n_{\beta}$ and the degree of internal two-cycle $d_{t}$. Since as mentioned before, there is a discrete axionic shift symmetry under $\operatorname{Im} T \rightarrow \operatorname{Im} T+N$ with $N$ being integer, we identify the axion as $\operatorname{Im} T$ and its decay constant as $f \simeq \sqrt{2 K_{T \bar{T}}} / 8 \pi^{2}$. The kinetic term including the instanton correction is extracted from the above Kähler potential,

$$
K_{T \bar{T}}=\operatorname{Re}\left(K_{T}\right)\left(\operatorname{Re}\left(K_{T}\right)+\frac{2}{T+\bar{T}}\right)+\operatorname{Im}\left(K_{T}\right)^{2},
$$

where

$$
\begin{aligned}
K_{T}= & -e^{K}\left[\frac{\kappa_{t t t}(T+\bar{T})^{2}}{2}-\sum_{n, d_{t}=1}^{\infty} \frac{d_{t}^{2} n_{\beta}(T+\bar{T})}{2 \pi n} e^{-\pi n d_{t}(T+\bar{T})} \cos \left(-i \pi n d_{t}(T-\bar{T})\right)\right. \\
& \left.+i \sum_{n, d_{t}=1}^{\infty} n_{\beta}\left(\frac{2 d_{t}^{2}(T+\bar{T})}{2 \pi n}+\frac{2 d_{t}}{(2 \pi)^{2} n^{2}}\right) e^{-\pi n d_{t}(T+\bar{T})} \sin \left(-i \pi n d_{t}(T-\bar{T})\right)\right] .
\end{aligned}
$$

In figure 1, we draw the quantum-corrected Kähler metric on the hypersurface $\operatorname{Im} T=0$ for single representative CY manifold, i.e., mirror quintic with $\chi=-200$ [40]. Now, we take into account the instanton effects up to order one hundred for mirror quintic by using the numerical code [46]. It is then found that the moduli Kähler metric has vanished at two points around the moduli value $\mathcal{O}(1)$ in the left panel in figure 1 , where the smaller modulus value leading to the vanishing Kähler metric satisfies

$$
K_{T}=0,
$$

whereas the larger one satisfies

$$
\begin{aligned}
\operatorname{Re}\left(K_{T}\right)+\frac{2}{T+\bar{T}} & =0, \\
\operatorname{Im} T & =0 .
\end{aligned}
$$

In this way, although the vanishing Kähler metric is unphysical, the tiny moduli Kähler metric is achieved at the values close to those of eqs. (3.63) or (3.64). It is then expected 
that the small axion decay constant is obtained around these points. Recall that, the negative instanton contributions in eq. (3.62) give rise to the small value of $K_{T}$ in general class of CY manifolds.

However, we have to take into account the fundamental domain where the large volume limit is applicable. To make the analysis concrete, we focus on the mirror quintic CY defined in eq. (3.40), where there are three special points such as orbifold, conifold, and LCS points, corresponding to $\psi=0,1, \infty$. The complex structure modulus $\psi$ in B-model is in one-to-one correspondence with Kähler modulus $T$ in the A-model under the mirror map [40]:

$$
T(\psi)=-\frac{5}{2 \pi}\left(\ln (5 \psi)-\frac{1}{w_{0}^{(1)}} \sum_{m=1}^{\infty} \frac{(5 m) !}{(m !)^{5}(5 \psi)^{5 m}}[\Psi(1+5 m)-\Psi(1+m)]\right)
$$

with $\Psi$ being the digamma function, and accordingly the quantum-corrected Yukawa coupling $\hat{\kappa}_{t t t}$ behaves in the limit $\psi \rightarrow 1$,

$$
\begin{aligned}
\hat{\kappa}_{t t t} & =5+\sum_{d_{t}=1}^{\infty} \frac{n_{d_{t}} d_{t}^{3} e^{-2 \pi d_{t} T}}{1-e^{-2 \pi d_{t} T}} \\
\propto & \frac{1}{(\psi-1)[-\ln (\psi-1)]^{3}}
\end{aligned}
$$

from which the Yukawa coupling diverges at the conifold point. Thus, the convergence radius in the large volume limit is determined so as to avoid such a singularity in the Yukawa coupling [40], i.e.,

$$
\operatorname{Re} T>\operatorname{Re} T(1) \simeq 1.208
$$

From this considerations, we conclude that the two points where the Kähler metric vanishes in the left panel in figure 1 are not in the large volume region, but in the conifold region. Although we find that these vanishing Kähler metric around the above similar points can be seen for the other one-parameter CY manifolds, the large volume expansion is not valid. Thus, the tiny axion decay constant is only obtained around the large volume point $\operatorname{Re} T \rightarrow \infty$ leading to $K_{T \bar{T}} \rightarrow 0$ as shown in the right panel in figure 1 , where the instanton corrections are enough suppressed. On the other hand, the trans-Planckian axion decay constant is not achieved in this mirror quintic CY. For other CY manifolds with negative small Euler characteristic, the large $\left|K_{T}\right|$ will lead to the trans-Planckian axion decay constant around the large volume point as discussed in ref. [18].

\subsubsection{Multiple axions}

We next extend the previous analysis to the multi moduli case in a similar step in section 3.3.1. The quantum-corrected Kähler potential for multi moduli $T^{i}$ with $i$ being the 

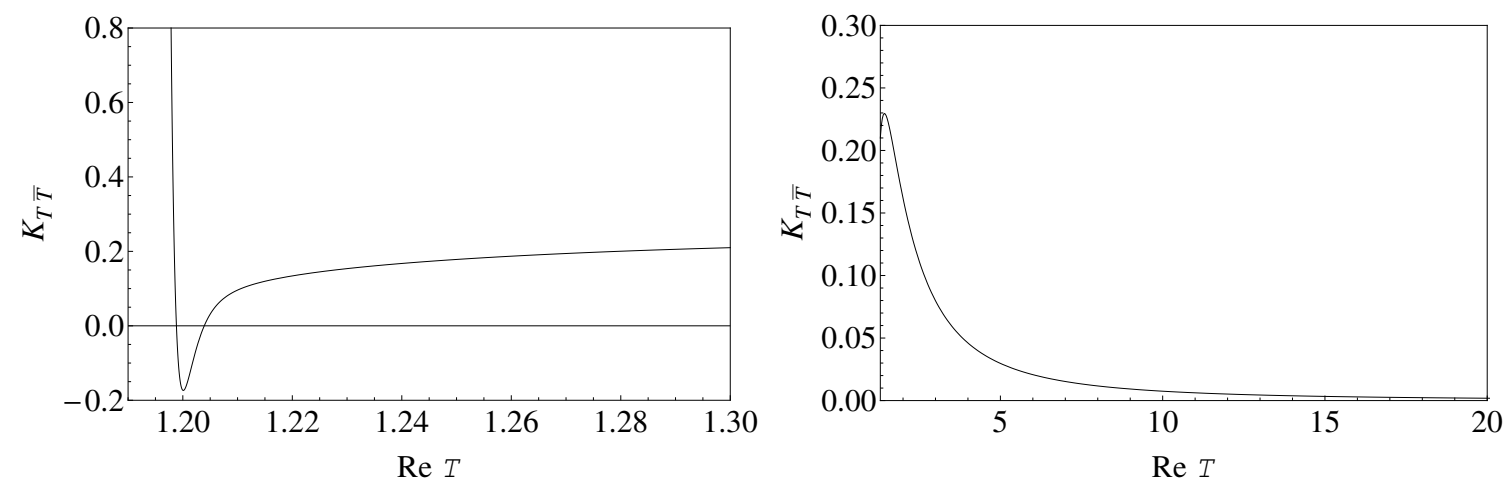

Figure 1. In both panels, the black curve corresponds to the quantum-corrected Kähler metric on the $\left(\operatorname{Re} T, K_{T \bar{T}}\right)$-plane for the mirror quintic CY manifold [40]. In the left (right) panel, the Kähler metric is plotted within the range, $1.18 \leq \operatorname{Re} T \leq 1.3(1.3 \leq \operatorname{Re} T \leq 20)$.

number of moduli fields is brought into the form [34, 35],

$$
\begin{aligned}
K(T, \bar{T})=-\ln [ & \frac{1}{6} \kappa_{i j k}\left(T^{i}+\bar{T}^{i}\right)\left(T^{j}+\bar{T}^{j}\right)\left(T^{k}+\bar{T}^{k}\right)-\frac{\zeta(3)}{4 \pi^{3}} \chi(\tilde{\mathcal{M}}) \\
& +\frac{2}{(2 \pi)^{2}} \sum_{\beta} \sum_{n=1}^{\infty} \frac{d_{i} n_{\beta}}{n^{2}}\left(T^{i}+\bar{T}^{i}\right) e^{-\pi n d_{k}\left(T^{k}+\bar{T}^{k}\right)} \cos \left(-i \pi n d_{j}\left(T^{j}-\bar{T}^{j}\right)\right) \\
& \left.+\frac{4}{(2 \pi)^{3}} \sum_{\beta} \sum_{n=1}^{\infty} \frac{n_{\beta}}{n^{3}} e^{-\pi n d_{k}\left(T^{k}+\bar{T}^{k}\right)} \cos \left(-i \pi n d_{j}\left(T^{j}-\bar{T}^{j}\right)\right)\right]
\end{aligned}
$$

around the large volume region, where the exponential terms show the instanton corrections labeled by the instanton number $n_{\beta}$ and the degree of internal two-cycles $d_{k}$. From the Kähler potential (3.68), we find that there are the discrete axionic shift symmetries, $\operatorname{Im} T^{i} \rightarrow \operatorname{Im} T^{i}+N$ with $N$ being integer. Thus, we identify the axions as $\operatorname{Im} T^{i}$ and the magnitude of these decay constant is proportional to $f \propto \operatorname{det}\left(K_{T^{i} \bar{T}^{j}}\right)$ due to the noncanonical kinetic terms of moduli fields. The moduli kinetic terms including the instanton correction become

$$
K_{T^{i} \bar{T}^{j}}=K_{T^{i}} K_{\bar{T}^{j}}-e^{K}\left[\kappa_{i j k}\left(T^{k}+\bar{T}^{k}\right)-\sum_{\beta} \sum_{n=1}^{\infty} \frac{n_{\beta} d_{i} d_{j}}{\pi n} e^{-\pi n d_{k}\left(T^{k}+\bar{T}^{k}\right)}\right],
$$

where

$$
\begin{aligned}
K_{T^{i}}= & -e^{K}\left[\frac{\kappa_{i j k}\left(T^{j}+\bar{T}^{j}\right)\left(T^{k}+\bar{T}^{k}\right)}{2}\right. \\
& -\sum_{\beta} \sum_{n=1}^{\infty} \frac{d_{i} d_{j} n_{\beta}\left(T^{j}+\bar{T}^{j}\right)}{2 \pi n} e^{-\pi n d_{k}\left(T^{k}+\bar{T}^{k}\right)} \cos \left(-i \pi n d_{k}\left(T^{k}-\bar{T}^{k}\right)\right) \\
& \left.+i \sum_{\beta} \sum_{n=1}^{\infty} n_{\beta}\left(\frac{2 d_{i} d_{j}\left(T^{j}+\bar{T}^{j}\right)}{2 \pi n}+\frac{2 d_{i}}{(2 \pi)^{2} n^{2}}\right) e^{-\pi n d_{k}\left(T^{k}+\bar{T}^{k}\right)} \sin \left(-i \pi n d_{k}\left(T^{k}-\bar{T}^{k}\right)\right)\right] .
\end{aligned}
$$


When all the moduli fields stay at the points satisfying

$$
K_{T^{i}}=0,
$$

or

$$
\begin{aligned}
\frac{T^{j}+\bar{T}^{j}}{2} K_{T^{j}}+1 & =0, \\
\operatorname{Im} T^{i} & =0,
\end{aligned}
$$

the moduli Kähler metrics satisfies the following equalities,

$$
\left(T^{j}+\bar{T}^{j}\right) K_{T^{i} \bar{T}^{j}}=0,
$$

from which, in both cases, the determinant of moduli Kähler metric vanishes simultaneously. This is because when the inverse matrix of moduli Kähler metric exists, all the moduli values are equal to zero at the points satisfying eqs. (3.71) or (3.72),

$$
T^{j}+\bar{T}^{j}=0,
$$

which contradict the large volume region of moduli space. As a result, at the certain moduli values satisfying eqs. (3.71) or (3.72), we obtain the vanishing determinant of moduli Kähler metric. Although the vanishing inverse Kähler metric is unphysical, we expect that the tiny determinant of moduli Kähler metric, i.e., tiny axion decay constant, is realized at the values close to those of eqs. (3.71) or (3.72).

However, when the Kähler metric around the points in eqs. (3.71) or (3.72) vanishes by the inclusion of instanton effects, the moduli values are determined by the cancellation between the classical and instanton contributions. It implies that, in a similar reason discussed in the previous section 3.3.1, these points are not in the large volume region, but in the conifold or other regions of special points. Thus, for the multiple axion cases, the tiny Kähler metric is only achieved around the large volume region $T^{i} \rightarrow \infty$ leading to $f \propto \operatorname{det}\left(K_{T^{i} \bar{T}^{j}}\right) \rightarrow 0$ in which the instanton corrections are enough suppressed. On the other hand, the trans-Planckian axion decay constant will be realized for certain class of CY manifold with negative small Euler characteristic [18].

\section{Massless axion and moduli stabilization}

As a result of the discussion presented so far, we have assumed that the moduli values are treated as parameters to obtain the tiny moduli Kähler metric, i.e., the tiny axion decay constant. In this section, we explore to stabilize the relevant complex structure modulus in type IIB string theory with an emphasis on the SCS point as discussed in section 3.1. To solve the strong CP problem and explain the current dark matter abundance, the massless axion with small axion decay constant and massive saxion (scalar partner of axion) are required at least at the compactification scale. The Planck data also favors the small axion decay constant in order not to overproduce the isocurvature perturbation [7]. As mentioned before, the couplings between our considered axions and gauge bosons are unrevealed for 
general CY background. Thus, in a way similar to the toroidal background [28, 29], we assume that the one-loop gauge kinetic function involves the axion associated with complex structure moduli.

To obtain the tiny decay constant of massless axion, we consider the moduli stabilization where the saxion $|z|$ is stabilized at the minimum satisfying $\partial_{z} K(|z|) \simeq 0$ by introducing the axion-independent uplifting sector rather than the superpotential of complex structure modulus $z$. In such a case, the axion becomes massless, since the Kähler potential is invariant under the monodromy transformation around the special point. We demonstrate the above scenario at the orbifold point of moduli space in section 3.1.1, for simplicity. For our purpose, we redefine the complex structure modulus $(h z)^{-\alpha_{1}}$ as $\varphi$ and then the Kähler potential is simplified as

$$
\begin{aligned}
K & =-\ln (-i(\tau-\bar{\tau}))+K(T+\bar{T})+K(|\varphi|), \\
W & =W(\tau, T),
\end{aligned}
$$

where $\tau$ and $T$ represent the axion-dilaton and Kähler moduli and $K(|\varphi|)$ corresponds to the Kähler potential in eq. (3.12) replaced by $\varphi$,

$$
K(|\varphi|)=-\ln \left[1+\frac{C_{2}}{C_{1}}|\varphi|^{-2+2 \alpha_{2} / \alpha_{1}}+\mathcal{O}\left(|\varphi|^{-2+2 \alpha_{3} / \alpha_{1}}\right)\right],
$$

with $C_{k}=\frac{A_{k}^{2}}{4 \pi^{6}} s_{k}^{3} c_{k} \kappa\left(-b s_{k}^{2}+2\left(\frac{2}{3} s_{k}^{2}+c_{k}^{2}\right)\right)$. Now, we perform a gauge transformation of the holomorphic three-form to achieve $K(|\varphi|) \rightarrow K(|\varphi|)+\ln \left|C_{1} \varphi\right|^{2}$ along with ref. [47],

$$
\Omega \rightarrow\left(C_{1} \varphi\right)^{-1} \Omega \text {. }
$$

In addition, we assume that the superpotential is non-perturbatively generated by the $D$ instanton or gaugino condensation on the hidden D7-branes wrapping on the internal cycle without depending on the complex structure modulus,

$$
W=W(\tau, T),
$$

where we consider the racetrack-type superpotential [48-51]. When the complex structure modulus appears in the superpotential, the axion (phase direction of $\varphi$ ) generically become massive. In this sense, we focus on the $\varphi$-independent superpotential to obtain the phenomenologically favorable light axion.

However, in the current setup, the supersymmetric minimum satisfying

$$
D_{I} W=W_{I}+K_{I} W=0
$$

where $W_{I}=\partial W / \partial \Phi^{I}$ and $K_{I}=\partial K / \partial \Phi^{I}$ with $\Phi^{I}$ being the axion-dilaton, Kähler and complex structure moduli, is not physical, since the Kähler metric of complex structure moduli vanishes at this minimum. In addition to the Kähler and superpotentials in eqs. (4.1) and (4.4), we thus introduce the uplifting sector such as anti D3-brane [52] around the large volume and small complex structure points of $\mathrm{CY}$ manifold, that is, $T \gg 1$ and $|\varphi| \ll 1$. The scalar potential is then characterized by the shift symmetric form [53, 54],

$$
V_{\text {up }}=e^{2 K / 3} \mathcal{P}(T+\bar{T},|\varphi|)
$$


where $\mathcal{P}$ is the $T$ - and $\varphi$-dependent function. The explicit form of $\mathcal{P}$ depends on the detailed D-brane setup.

From the scalar potential in four-dimensional $\mathcal{N}=1$ supergravity,

$$
V=V_{F}+V_{\mathrm{up}}
$$

with

$$
V_{F}=e^{K}\left(K^{I \bar{J}} D_{I} W D_{\bar{J}} \bar{W}-3|W|^{2}\right),
$$

we find the local minimum of the complex structure modulus. The extremal condition of $\varphi$ is yielded by

$$
\partial_{\varphi} V \simeq m_{3 / 2}^{2}\left[K_{\bar{\varphi}} \partial_{\varphi}\left(K^{\varphi \bar{\varphi}} K_{\varphi}\right)+3 \partial_{\varphi}(\ln \mathcal{P})\right]=0
$$

where $m_{3 / 2}=e^{\langle K\rangle / 2}\langle W\rangle$ is the gravitino mass. Now, we employ the condition of almost vanishing cosmological constant $\langle V\rangle \simeq 0$ and $\left\langle V_{\mathrm{up}}\right\rangle \simeq 3 m_{3 / 2}^{2}$ which is valid for the wellknown Kähler moduli stabilization, e.g., Kachru-Kallosh-Linde-Trivedi (KKLT) [52] or racetrack scenario [48-51].

Guided by the Kähler potential in eq. (4.1), we obtain the following equality,

$$
K_{\bar{\varphi}} \partial_{\varphi}\left(K^{\varphi \bar{\varphi}} K_{\varphi}\right) \simeq-\left(\frac{C_{2}}{C_{1}}\right)^{2}\left(\frac{\alpha_{2}}{\alpha_{1}}-1\right)|\varphi|^{4\left(\frac{\alpha_{2}}{\alpha_{1}}-1\right)} \varphi^{-1}
$$

and consequently the vacuum expectation value of complex structure modulus is determined to satisfy the extremal condition (4.9),

$$
|\varphi|^{4 \frac{\alpha_{2}}{\alpha_{1}}-5} \simeq 3\left(\frac{C_{1}}{C_{2}}\right)^{2}\left(\frac{\alpha_{2}}{\alpha_{1}}-1\right)\left|\partial_{\varphi}(\ln \mathcal{P})\right|
$$

where $\alpha_{2} / \alpha_{1}$ is grater than unity in our examples in table 1 with distinct $\alpha_{i}$, and the saxion $|\varphi|$ is only stabilized at the above minimum by the axion-independent scalar potential. Now, we assume that the mass squared of saxion is positive, since they depend on the second derivative of $\partial_{\varphi} \partial_{\bar{\varphi}}(\ln \mathcal{P})$. The other closed string moduli can be stabilized at the vacuum by the superpotential.

In this way, it is possible to obtain the massless axion along the above moduli stabilization procedure. From the formula of axion Kähler metric,

$$
K_{\theta \theta}=K_{\varphi \bar{\varphi}}|\varphi|^{2} \simeq-\left(\frac{\alpha_{2}}{\alpha_{1}}-1\right)^{2}\left(\frac{C_{2}}{C_{1}}\right)|\varphi|^{2\left(\frac{\alpha_{2}}{\alpha_{1}}-1\right)},
$$

and the vacuum expectation value of $|\varphi|$ in eq. (4.11), the tiny Kähler metric of axion $\theta=\arg (\varphi)$, i.e., the tiny axion decay constant, is achieved only when $\partial_{\varphi}(\ln \mathcal{P})$ is much smaller than unity. For example, the cosmologically favorable axion Kähler metric $\sqrt{2 K_{\theta \theta}} \simeq 10^{12} \mathrm{GeV}$ is achieved under $\partial_{\varphi}(\ln \mathcal{P}) \simeq 3 \times 10^{-7}$ for the CY manifold defined in $\mathbb{P}_{3,2,2,1,1,1}^{5}[4,6]$. In a similar fashion, we can realize the suppressed axion decay constant at the other SCS points and conifold point. Note that the Kähler metric is governed by the vacuum expectation value of $\partial_{\varphi}(\ln \mathcal{P})=\partial_{\varphi} \mathcal{P} / \mathcal{P}$, whereas the cosmological constant is 
dominated by that of $\mathcal{P}$. Therefore, the obtained small axion Kähler metric is compatible with the tiny cosmological constant under $\partial_{\varphi} \mathcal{P} \ll \mathcal{P}$. However, it is interesting and challenging issue to obtain such a small value of $\partial_{\varphi}(\ln \mathcal{P})$, since its specific form depends on the concrete D-brane setup taking into account the Ramond-Ramond tadpole condition. We study the detailed construction of tiny axion decay constant elsewhere.

\section{Conclusion}

In this paper, we have studied the detail of the quantum and geometrical corrections for a decay constant of closed string axion with an emphasis on the special points of PicardFuchs equation, in particular the small complex structure point in section 3.1, conifold point in section 3.2, and large complex structure (large volume) point in section 3.3, around which the axion particles naturally appear in the low-energy effective theory. The axionic shift symmetries of the Kähler potential are originating from the geometrical symmetries around the special points. Furthermore, in the type IIB string theory, the decay constant of axion associated with the complex structure modulus is irrelevant with the string scale in comparison with that of Kähler modulus. It is thus interesting to discuss the axion associated with complex structure modulus.

On the basis of topological string theory, we find the general expression of period vector and its monodromy transformation for typical one-parameter CY manifolds in table 1 by solving the corresponding Picard-Fuchs differential equations. It then turns out that only the tiny axion decay constant can be realized around the special points, since the axion Kähler metric exactly vanishes at all the special points. We also demonstrate the moduli stabilization to realize such a situation in section 4. It is interesting to embed our scenario in the detailed D-brane setup and reveal the couplings between the axion and matter fields in the visible sector, which will be studied elsewhere.

\section{Acknowledgments}

The authors thank H. Abe for valuable comments. H.O. would like to thank K. Choi, R. Gopakumar, T. Higaki and G. Shiu for useful discussions. A.O. and H.O. are grateful to Y. Honma for fruitful discussions. A.O. and H.O. also thank the Yukawa Institute for Theoretical Physics at Kyoto University. Discussions during the YITP workshop YITPW-16-05 "Strings and Fields 2016" were useful to complete this work.

Open Access. This article is distributed under the terms of the Creative Commons Attribution License (CC-BY 4.0), which permits any use, distribution and reproduction in any medium, provided the original author(s) and source are credited.

\section{References}

[1] R.D. Peccei and H.R. Quinn, CP Conservation in the Presence of Instantons, Phys. Rev. Lett. 38 (1977) 1440 [INSPIRE]. 
[2] J. Preskill, M.B. Wise and F. Wilczek, Cosmology of the Invisible Axion, Phys. Lett. B 120 (1983) 127 [INSPIRE].

[3] L.F. Abbott and P. Sikivie, A Cosmological Bound on the Invisible Axion, Phys. Lett. B 120 (1983) 133 [INSPIRE].

[4] M. Dine and W. Fischler, The Not So Harmless Axion, Phys. Lett. B 120 (1983) 137 [INSPIRE].

[5] Kamiokande-II collaboration, K. Hirata et al., Observation of a Neutrino Burst from the Supernova SN 1987a, Phys. Rev. Lett. 58 (1987) 1490 [INSPIRE].

[6] R.M. Bionta et al., Observation of a Neutrino Burst in Coincidence with Supernova SN 1987a in the Large Magellanic Cloud, Phys. Rev. Lett. 58 (1987) 1494 [InSPIRE].

[7] Planck collaboration, P.A.R. Ade et al., Planck 2015 results. XX. Constraints on inflation, Astron. Astrophys. 594 (2016) A20 [arXiv: 1502.02114] [InSPIRE].

[8] J.E. Kim, Light Pseudoscalars, Particle Physics and Cosmology, Phys. Rept. 150 (1987) 1 [INSPIRE].

[9] K. Freese, J.A. Frieman and A.V. Olinto, Natural inflation with pseudo-Nambu-Goldstone bosons, Phys. Rev. Lett. 65 (1990) 3233 [INSPIRE].

[10] K. Choi and J.E. Kim, Harmful Axions in Superstring Models, Phys. Lett. B 154 (1985) 393 [Erratum ibid. B 156 (1985) 452] [INSPIRE].

[11] T. Banks, M. Dine, P.J. Fox and E. Gorbatov, On the possibility of large axion decay constants, JCAP 06 (2003) 001 [hep-th/0303252] [INSPIRE].

[12] P. Svrček and E. Witten, Axions In String Theory, JHEP 06 (2006) 051 [hep-th/0605206] [INSPIRE].

[13] J.P. Conlon, The QCD axion and moduli stabilisation, JHEP 05 (2006) 078 [hep-th/0602233] [INSPIRE].

[14] V. Balasubramanian, P. Berglund, J.P. Conlon and F. Quevedo, Systematics of moduli stabilisation in Calabi-Yau flux compactifications, JHEP 03 (2005) 007 [hep-th/0502058] [INSPIRE].

[15] K. Dasgupta, H. Firouzjahi and R. Gwyn, On The Warped Heterotic Axion, JHEP 06 (2008) 056 [arXiv: 0803.3828] [INSPIRE].

[16] E.I. Buchbinder, A. Constantin and A. Lukas, Heterotic QCD axion, Phys. Rev. D 91 (2015) 046010 [arXiv: 1412.8696] [INSPIRE].

[17] G. Honecker and W. Staessens, On axionic dark matter in Type IIA string theory, Fortsch. Phys. 62 (2014) 115 [arXiv:1312.4517] [InSPIRE].

[18] J.P. Conlon and S. Krippendorf, Axion decay constants away from the lamppost, JHEP 04 (2016) 085 [arXiv : 1601.00647] [INSPIRE].

[19] H. Abe, T. Kobayashi and H. Otsuka, Towards natural inflation from weakly coupled heterotic string theory, PTEP 2015 (2014) 063E02 [arXiv: 1409.8436] [INSPIRE].

[20] H. Abe, T. Kobayashi and H. Otsuka, Natural inflation with and without modulations in type IIB string theory, JHEP 04 (2015) 160 [arXiv: 1411.4768] [INSPIRE].

[21] J.E. Kim, H.P. Nilles and M. Peloso, Completing natural inflation, JCAP 01 (2005) 005 [hep-ph/0409138] [INSPIRE]. 
[22] D. Gepner, Exactly Solvable String Compactifications on Manifolds of SU(N) Holonomy, Phys. Lett. B 199 (1987) 380 [INSPIRE].

[23] D. Gepner, Space-Time Supersymmetry in Compactified String Theory and Superconformal Models, Nucl. Phys. B 296 (1988) 757 [inSPIRE].

[24] I. García-Etxebarria, T.W. Grimm and I. Valenzuela, Special Points of Inflation in Flux Compactifications, Nucl. Phys. B 899 (2015) 414 [arXiv:1412.5537] [inSPIRE].

[25] T. Kobayashi, A. Oikawa and H. Otsuka, New potentials for string axion inflation, Phys. Rev. D 93 (2016) 083508 [arXiv:1510.08768] [INSPIRE].

[26] K. Akita, T. Kobayashi, A. Oikawa and H. Otsuka, Moduli mediation without moduli-induced gravitino problem, JHEP 05 (2016) 178 [arXiv:1603.08399] [INSPIRE].

[27] T.W. Grimm and J. Louis, The effective action of $N=1$ Calabi-Yau orientifolds, Nucl. Phys. B 699 (2004) 387 [hep-th/0403067] [INSPIRE].

[28] D. Lüst and S. Stieberger, Gauge threshold corrections in intersecting brane world models, Fortsch. Phys. 55 (2007) 427 [hep-th/0302221] [inSPIRE].

[29] R. Blumenhagen, B. Körs, D. Lüst and S. Stieberger, Four-dimensional String Compactifications with D-branes, Orientifolds and Fluxes, Phys. Rept. 445 (2007) 1 [hep-th/0610327] [INSPIRE].

[30] E. Witten, Topological $\sigma$-models, Commun. Math. Phys. 118 (1988) 411 [INSPIRE].

[31] E. Witten, Mirror manifolds and topological field theory, hep-th/9112056 [INSPIRE].

[32] S. Gukov, C. Vafa and E. Witten, CFT's from Calabi-Yau four folds, Nucl. Phys. B 584 (2000) 69 [Erratum ibid. B 608 (2001) 477] [hep-th/9906070] [INSPIRE].

[33] S.B. Giddings, S. Kachru and J. Polchinski, Hierarchies from fluxes in string compactifications, Phys. Rev. D 66 (2002) 106006 [hep-th/0105097] [INSPIRE].

[34] S. Hosono, A. Klemm, S. Theisen and S.-T. Yau, Mirror symmetry, mirror map and applications to Calabi-Yau hypersurfaces, Commun. Math. Phys. 167 (1995) 301 [hep-th/9308122] [INSPIRE].

[35] S. Hosono, A. Klemm, S. Theisen and S.-T. Yau, Mirror symmetry, mirror map and applications to complete intersection Calabi-Yau spaces, Nucl. Phys. B 433 (1995) 501 [hep-th/9406055] [INSPIRE].

[36] B.R. Greene and C.I. Lazaroiu, Collapsing D-branes in Calabi-Yau moduli space. 1., Nucl. Phys. B 604 (2001) 181 [hep-th/0001025] [INSPIRE].

[37] Y. Yang Y.-H. Chen and N. Yui, Monodromy of Picard-Fuchs differential equations for Calabi-Yau threefolds, math/0605675.

[38] A. Erdelyi ed., The Bateman Project, Higher Transcendental Functions, Vol. 1, Sec. 5.3.-5.6, McGraw-Hill Book Company, New York, U.S.A. (1953).

[39] A. Ceresole, R. D'Auria, S. Ferrara and A. Van Proeyen, Duality transformations in supersymmetric Yang-Mills theories coupled to supergravity, Nucl. Phys. B 444 (1995) 92 [hep-th/9502072] [INSPIRE].

[40] P. Candelas, X.C. De La Ossa, P.S. Green and L. Parkes, A pair of Calabi-Yau manifolds as an exactly soluble superconformal theory, Nucl. Phys. B 359 (1991) 21 [INSPIRE]. 
[41] A. Klemm and S. Theisen, Considerations of one modulus Calabi-Yau compactifications: Picard-Fuchs equations, Kähler potentials and mirror maps, Nucl. Phys. B 389 (1993) 153 [hep-th/9205041] [INSPIRE].

[42] C.I. Lazaroiu, Collapsing D-branes in one parameter models and small / large radius duality, Nucl. Phys. B 605 (2001) 159 [hep-th/0002004] [INSPIRE].

[43] A. Hebecker and J. March-Russell, The ubiquitous throat, Nucl. Phys. B 781 (2007) 99 [hep-th/0607120] [INSPIRE].

[44] H.L. Verlinde, Holography and compactification, Nucl. Phys. B 580 (2000) 264 [hep-th/9906182] [INSPIRE].

[45] M.-x. Huang, A. Klemm and S. Quackenbush, Topological string theory on compact Calabi-Yau: Modularity and boundary conditions, Lect. Notes Phys. 757 (2009) 45 [hep-th/0612125] [INSPIRE].

[46] A. Klemm, Instanton.

[47] A. Giryavets, S. Kachru, P.K. Tripathy and S.P. Trivedi, Flux compactifications on Calabi-Yau threefolds, JHEP 04 (2004) 003 [hep-th/0312104] [INSPIRE].

[48] N.V. Krasnikov, On Supersymmetry Breaking in Superstring Theories, Phys. Lett. B 193 (1987) 37 [INSPIRE].

[49] T.R. Taylor, Dilaton, gaugino condensation and supersymmetry breaking, Phys. Lett. B 252 (1990) 59 [INSPIRE].

[50] J.A. Casas, Z. Lalak, C. Muñoz and G.G. Ross, Hierarchical Supersymmetry Breaking and Dynamical Determination of Compactification Parameters by Nonperturbative Effects, Nucl. Phys. B 347 (1990) 243 [inSPIRE].

[51] B. de Carlos, J.A. Casas and C. Muñoz, Supersymmetry breaking and determination of the unification gauge coupling constant in string theories, Nucl. Phys. B 399 (1993) 623 [hep-th/9204012] [INSPIRE].

[52] S. Kachru, R. Kallosh, A.D. Linde and S.P. Trivedi, de Sitter vacua in string theory, Phys. Rev. D 68 (2003) 046005 [hep-th/0301240] [INSPIRE].

[53] K. Choi, A. Falkowski, H.P. Nilles, M. Olechowski and S. Pokorski, Stability of flux compactifications and the pattern of supersymmetry breaking, JHEP 11 (2004) 076 [hep-th/0411066] [INSPIRE].

[54] K. Choi, A. Falkowski, H.P. Nilles and M. Olechowski, Soft supersymmetry breaking in KKLT flux compactification, Nucl. Phys. B 718 (2005) 113 [hep-th/0503216] [INSPIRE]. 